\title{
Fast-ion pressure dominating the mass dependence of the core heat transport in ASDEX Upgrade H-modes
}

P. A. Schneider ${ }^{1}$, N. Bonanomi ${ }^{1}$, C. Angioni ${ }^{1}$, M. Weiland ${ }^{1}$, M. Cavedon $^{1}$, P. David ${ }^{1}$, R. Fischer $^{1}$, P. Hennequin ${ }^{2}$, J. Hobirk ${ }^{1}$, A. Kappatou ${ }^{1}$, B. Kurzan ${ }^{1}$, R. M. McDermott ${ }^{1}$, U. Plank ${ }^{1}$, T. Pütterich ${ }^{1}$, F. Ryter ${ }^{1}$, M. Willensdorfer ${ }^{1}$, the ASDEX Upgrade Team ${ }^{\text {a }}$ and the EUROfusion MST1 Team ${ }^{\mathrm{b}}$

${ }^{1}$ Max-Planck-Institut für Plasmaphysik, Boltzmannstr. 2, 85748 Garching, Germany

${ }^{2}$ Laboratoire de Physique des Plasmas, CNRS UMR7648, Ecole Polytechnique, 91128 Palaiseau,

France

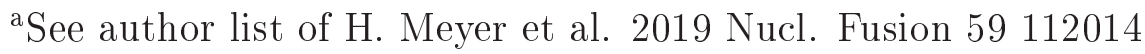

${ }^{\mathrm{b}}$ See author list of B. Labit et al. 2019 Nucl. Fusion 59086020

Abstract:

H-mode plasmas in ASDEX Upgrade (AUG) using different hydrogen isotopes are analysed with respect to their core transport properties. The experimental results are discussed and we present gyrokinetic simulations which are able to reproduce the experimental observations.

A novel strategy allows us to disentangle core and pedestal physics by mitigating the isotopic dependence of pedestal properties while keeping the heat and particle sources the same. Matched pedestal profiles are obtained between hydrogen $(\mathrm{H})$ and deuterium $(\mathrm{D})$ plasmas when increasing the triangularity in $\mathrm{H}$ plasmas with respect to $\mathrm{D}$ plasmas.

In the core of these plasmas little isotopic dependence is observed when the fast-ion content is low $W_{\text {fast }} / W_{\text {th }}<1 / 3$. Quasi-linear modelling with TGLF reproduces the experimental trends under these conditions. For larger fast-ion fractions an isotope dependence is observed in the core heat transport. This is related to a difference in fast-ion stabilization of turbulent transport. The fast-ion pressure in $\mathrm{H}$ and $\mathrm{D}$ plasmas is different due to the mass dependence in the fastion slowing down time as well as to operational restrictions when heating with $\mathrm{H}$ neutral beam injection (H-NBI) or D-NBI. Typically, $W_{\text {fast }, \mathrm{H}}<1 / 2 W_{\mathrm{fast}, \mathrm{D}}$ for comparable NBI heating powers in AUG.

The gyrokinetic analysis shows that linear growth rates of ITG modes do not show a pure gyroBohm mass dependence, but follow the experimentally observed mass dependence when taking collisions, EM-effects and fast ions into account. Non-linear gyrokinetic simulations reproduce the experimental heat fluxes for different isotopes when fast ions are included. This highlights the role of the fast-ion pressure as a key element to explain the observed differences in the core of $\mathrm{H}$ and D plasmas.

\section{Introduction}

Accurate knowledge of particle and energy confinement is of paramount relevance for the extrapolation to a fusion reactor. The bulk of experimental data in tokamak research is obtained in deuterium (D) plasmas for which the predictive capability has improved significantly over recent years. In contrast, the mass dependence of confinement still challenges the current theoretical understanding. This is true for mixed deuterium and tritium plasmas needed for fusion, but also for hydrogen $(\mathrm{H})$ plasmas. Experience developed during $\mathrm{H}$ operation is required for a successful 
operation in D and DT, as transferring knowledge from the pre-nuclear phase to the nuclear phase is an essential step for ITER and any future nuclear fusion plant.

In H-mode plasmas there are two major contributions to energy confinement: the heat and particle transport in the core and the stability of the edge transport barrier. For simplicity they are often treated independently. However, recent experimental and theoretical studies showed that they can be strongly coupled in tokamak [1,2] as well as in stellarator [3] plasmas. This is particularly important for isotope studies.

In electron heated L-modes in ASDEX Upgrade (AUG) the mass dependence in the equipartition is found to yield different confinement in $\mathrm{H}$ and $\mathrm{D}$, because the ion heat transport is more stiff than the electron heat transport [4]. This is similarly observed in ohmic plasmas in JET and FT-2 [5,6]. NBI-heated identity L-modes (matched $\rho_{\star}, \nu_{\star}, \beta$ and $q$ ) in JET are dominated by high stiffness in the core transport and in this regime the confinement scale invariance principle is satisfied [7]. In the edge of AUG and JET-ILW L-mode plasmas drift wave turbulence is strongly influenced by the parallel electron dynamics and identified as a candidate to explain the reduced heat and particle transport in $\mathrm{D}$ compared to $\mathrm{H}$ [8] although due to the overall poor particle confinement in L-mode the impact on the core plasma is negligible. This changes for H-modes where reduced particle confinement is observed in the pedestal region of AUG and the gas puff has to be increased by an order of magnitude in $\mathrm{H}$ to match pedestal top densities of $\mathrm{D}[9]$. Reduced particle confinement with $\mathrm{H}$ is also found in JET-ILW where the electron density $n_{\mathrm{e}} \propto A^{0.57 \pm 0.05}$ with the mass number $A$ is reported [10. In JT60-U a mass dependence of the critical gradient for turbulent transport is observed in the core and it was suggested $\beta$ stabilisation of the edge pedestal could results in a bootstrap like enhancement of confinement in D [11].

Higher fast-ion content in D is pointed out in the JT60-U study, which complements the edge stabilisation effect via a higher contribution to the total pressure. The fast ions themselves can also have a direct impact on the turbulence stabilisation and introduce a mass dependence as observed in JET-ILW L-modes [12. Additionally, there are other factors, such as $E \times B$ shear flows or $T_{\mathrm{e}} / T_{\mathrm{i}}$ 13, 14] or main ion dilution by fast ions [15, which can play a role when the heating mix is changed. A change of the heating mix is difficult to avoid when the first priority is to maintain isotope purity or when the heat and particle sources are varied to compensate for the edge isotope dependencies. Clear comparisons with the theoretical predictions are hindered by the difficulties separating the roles of individual contributions.

In this paper we present a new approach to conduct the experiments which greatly reduces the complexity of codependent processes interacting with each other. It allows us for the first time to experimentally separate the effects of core transport and the edge pedestal with respect to the mass dependence. So compared to previous studies in H-mode this allows to draw conclusions for the core heat transport without the necessity of integrated core-edge modelling. This decoupling is achieved by comparing differently shaped plasmas, with a higher triangularity $\delta$ at the edge in $\mathrm{H}$ than in D. The higher $\delta$ compensates for the loss of particle confinement in $\mathrm{H}$ and a pedestal match between $\mathrm{H}$ and $\mathrm{D}$ is achieved with heat and particle sources being nearly identical. It has to be pointed out that all plasmas still have $\delta<0.4$, so they are not comparable to extremely shaped 
plasmas where a strong stabilisation of the edge pressure has been observed as e.g. in DIII-D [16]. Because of the multi-layered nature of the physics processes involved, we decided to separate our report into two parts. The one presented here will focus on the core transport phenomena taking advantage of the matched pedestal properties. A separate publication will discuss in detail the edge physics and, in particular, propose an explanation on how the pedestal is matched with different triangularities as well on how parameter changes such as the gas fuelling level will result in a strong deviation dependent on mass number.

The paper begins with an overview of the experimental setup. In section 2.1 we will assess the parameters necessary to mitigate the influence of the ion mass on the pedestal. This allows to select a set of discharges with similar pedestal properties which are suited for the core transport analysis. For this subset we then present the plasma parameters important for a discussion of the transport physics in section 2.2. These parameters include the electron density $n_{\mathrm{e}}$, electron temperature $T_{\mathrm{e}}$, ion temperature $T_{\mathrm{i}}$, toroidal rotation $v_{\text {tor }}$ and fast-ion density $n_{\text {fast }}$. We will discuss the differences between hydrogen and deuterium plasmas and perform a power balance analysis highlighting the differences and similarities in core transport.

To quantify the impact of parameter variations other than the isotope mass, i.e. $v_{\text {tor }}, n_{\text {fast }}$ and differences in the equilibrium and heat deposition, we utilize the quasilinear TGLF model 17, 18] within the transport code ASTRA [19, 20] to compare our observations against theoretical predictions. For high power cases, where $n_{\text {fast,H }} \ll n_{\text {fast,D }}$, the impact of the non-linear turbulence stabilisation by fast ions is quantified with gyrokinetic simulations using the Gene code [21,22]. We conclude with a discussion of the experimental and theoretical observations.

\section{Experiments}

The plasma scenario used in ASDEX Upgrade (AUG) has a plasma current of $I_{\mathrm{p}}=0.8 \mathrm{MA}$ and a toroidal magnetic field $B_{\mathrm{t}}=-2.5 \mathrm{~T}$ - with $B_{\mathrm{t}}<0$ corresponding to a counter current field with favourable $\nabla B$ drift direction - and run with hydrogen as well as deuterium as main ion species. The engineering parameters which are varied are plasma shape, auxiliary heating power and gas fuelling. In Table 1 all the discharges and time intervals used in this paper are listed, these plasma phases will be referred to by their ID listed in the table. All data is ELM synchronised and only pre-ELM profiles are shown. Not all parameters can be varied at the same time, in particular, high heating power is incompatible with low gas fuelling for hydrogen. The reason for this is the longer mean free path of hydrogen beam neutrals resulting in higher beam shine through which triggers the heat shield protection of AUG. The impurity content is relatively low in all plasmas and we find the effective charge number $Z_{\mathrm{eff}}$ around 1.5 or lower, the value of $Z_{\mathrm{eff}}$ as measured via the bremsstrahlung spectrum is taken into account in the analysis.

The first set of parameter scans is done at moderate heating power with $P_{\mathrm{NBI}}=4.5 \mathrm{MW}$ and $P_{\mathrm{ECRH}}=3.4 \mathrm{MW}$, resulting in a $P_{\text {tot }}=P_{\text {heat }}-P_{\text {rad }} \sim 7 \mathrm{MW}$. It has to be noted that for $\mathrm{H}$ and $\mathrm{D}$ we utilize H-NBI and D-NBI respectively. The implications following this choice are discussed in section 2.2.4 and 2.2.5. The scans are mainly 2-point parameter variations. For gas puffing rates $\Gamma$ 


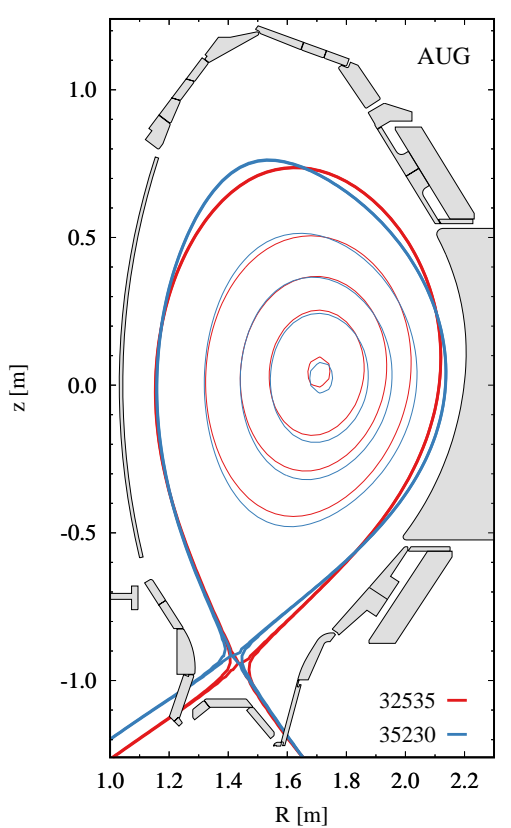

FIG. 1: Flux surfaces $\left(\rho_{\mathrm{pol}}=[0.1,0.4,0.6,0.8,1.0]\right)$ of plasmas with different triangularity 32535 with $\delta=0.22$ and 35230 with $\delta=0.37$.

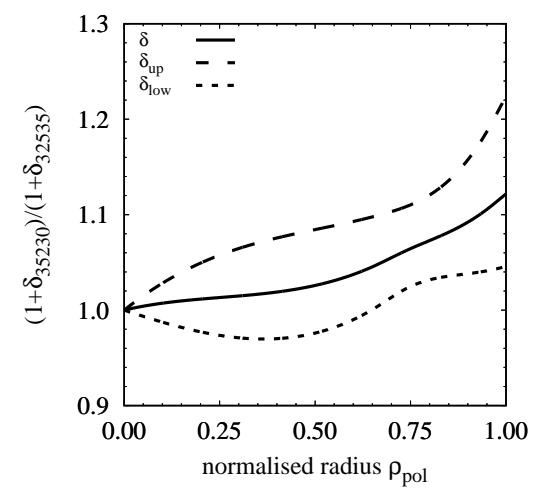

FIG. 2: Radial profile of the relative difference in triangularity for plasmas with an average triangularity at the edge of $\delta=0.22$ and $\delta=0.37$. 
this means $\Gamma_{\text {low }}=1 \cdot 10^{21}$ electrons $/ \mathrm{s}$ is compared to $\Gamma_{\text {high }}=8 \cdot 10^{21}$ electrons $/ \mathrm{s}$. In this range the deuterium plasmas exhibit an offset linear correlation between gas flux and density as described in detail in the next section. The gas puff range discussed here is low compared to detachment or heat exhaust studies which would use $\Gamma_{\text {high }}=20-40 \cdot 10^{21}$ electrons/s in AUG. For the average plasma triangularity $\delta=\left(\delta_{\mathrm{up}}+\delta_{\text {low }}\right) / 2.0$ the low value is around 0.22 and the high value 0.37 , this difference is illustrated in figure 1 The triangularity of individual flux surfaces is most different at the plasma edge $\rho_{\text {pol }}>0.8$ - the quoted values are calculated for the separatrix - and becomes smaller towards the plasma core which is illustrated for the upper and lower triangularity separately in figure 2, $\rho_{\text {pol }}$ is the normalized radius with $\rho_{\text {pol }}=\sqrt{\left(\Psi-\Psi_{\text {axis }}\right) /\left(\Psi_{\text {sep }}-\Psi_{\text {axis }}\right)}, \Psi$ being the poloidal magnetic flux and $\Psi_{\text {axis }}, \Psi_{\text {sep }}$ the flux at the magnetic axis and the separatrix. The strike lines cannot always be matched when comparing differently shaped plasmas. However, in the relevant range the strike line position has negligible impact on the density in AUG which was confirmed in a dedicated scan of the divertor configuration in L-mode. The main ions are either deuterium or hydrogen, with about $2-10 \%$ minority of the other species. These three parameter variations result in 8 different combinations at moderate heating power.

For detailed core transport analysis and modelling we focus on a subset of these parameter scans. This subset contains discharges with matched pedestal, i.e. low triangularity in D and high triangularity in $\mathrm{H}$. We discuss $\mathrm{H}$, D pairs with matched rotation and different fast-ion content and with matched fast-ion content and different rotation.

The second set is at higher heating power and higher fast-ion fraction than before to test the impact of $n_{\text {fast }}$ on the transport. The heating power is $P_{\mathrm{NBI}}=9.2 \mathrm{MW}$ and $P_{\mathrm{ECRH}}=2.2 \mathrm{MW}$ for hydrogen. The main ion mass is changed simultaneously with the triangularity. Meaning a high $\delta$ hydrogen plasma is compared with a low $\delta$ deuterium plasma.

In the remainder of this section we will describe the consequences these changes have on individual plasma properties like density and temperature. The diagnostics used are electron cyclotron emission ECE [23] for electron temperature, Thomson scattering [24] for electron temperature and density, Li-Beam [25] for edge density, charge exchange recombination spectroscopy CXRS [26, 27] for ion temperatures and rotation, bolometers [28] for radiation profiles, as well as fast-ion $\mathrm{D}_{\alpha}$ FIDA [29] for fast-ion densities.

\subsection{Edge pedestal}

\section{Electron density:}

The typical density behaviour in AUG with different isotopes is illustrated in figure 3. For low gas puff levels the edge density in $\mathrm{H}$ is $n_{\mathrm{e}, \mathrm{H}} \sim(0.8-0.9) n_{\mathrm{e}, \mathrm{D}}$. The density of a $\mathrm{D}$ plasma can be increased via a variation of the particle source. For AUG there is an offset linear density response for divertor fuelling between $1-8 \cdot 10^{21}$ particles/s. For higher fuelling rates non-linear effects like detachment and the formation of a high field side high density front [30] will result in a more complicated relation between gas puffing and particle sources in the main plasma. With low triangularity little correlation between density and fuelling is observed for $\mathrm{H}$ plasmas. At a gas puff level of $8 \cdot 10^{21} / \mathrm{s}$ we find $n_{\mathrm{e}, \mathrm{H}} \sim 0.7 n_{\mathrm{e}, \mathrm{D}}$, indicating a substantial difference between $\mathrm{H}$ 


\begin{tabular}{|c|c|c|c|c|c|c|c|}
\hline shot & $\begin{array}{l}\text { time } \\
{[\mathrm{s}]}\end{array}$ & isotope & $\begin{array}{r}P_{\text {tot }} \\
\text { [MW] }\end{array}$ & $\begin{array}{c}\Gamma \\
{\left[10^{21} / \mathrm{s}\right]}\end{array}$ & $\delta$ & $W_{\text {fast }} / W_{\text {th }}$ & ID \\
\hline 32535 & $4.2-4.5$ & $\mathrm{D}$ & 6.6 & 2.0 & 0.22 & 0.28 & 1 \\
\hline 34716 & $1.6-2.0$ & $\mathrm{H}$ & 6.8 & 8.0 & 0.22 & 0.14 & 2 \\
\hline 34796 & $3.8-4.2$ & $\mathrm{D}$ & 9.5 & 9.9 & 0.25 & 0.37 & 3 \\
\hline 35230 & $2.4-3.0$ & $\mathrm{H}$ & 6.9 & 1.0 & 0.22 & 0.15 & 4 \\
\hline 35230 & $4.2-4.8$ & $\mathrm{H}$ & 7.0 & 1.0 & 0.37 & 0.10 & 5 \\
\hline 35231 & $2.7-3.0$ & $\mathrm{H}$ & 6.7 & 8.2 & 0.35 & 0.07 & 6 \\
\hline 35275 & $4.0-4.7$ & $\mathrm{H}$ & 10.4 & 6.7 & 0.37 & 0.14 & 7 \\
\hline 35852 & $2.2-2.6$ & $\mathrm{D}$ & 7.0 & 0.9 & 0.26 & 0.15 & 8 \\
\hline 35852 & $4.0-4.4$ & $\mathrm{D}$ & 7.3 & 7.6 & 0.25 & 0.13 & 9 \\
\hline 35852 & 5.3-5.6 & $\mathrm{D}$ & 7.2 & 6.9 & 0.36 & 0.11 & 10 \\
\hline 35852 & $7.6-8.0$ & $\mathrm{D}$ & 7.0 & 0.9 & 0.37 & 0.15 & 11 \\
\hline
\end{tabular}

TABLE I: Discharges AND TIME intervals USED in THIS PUBliCATION.

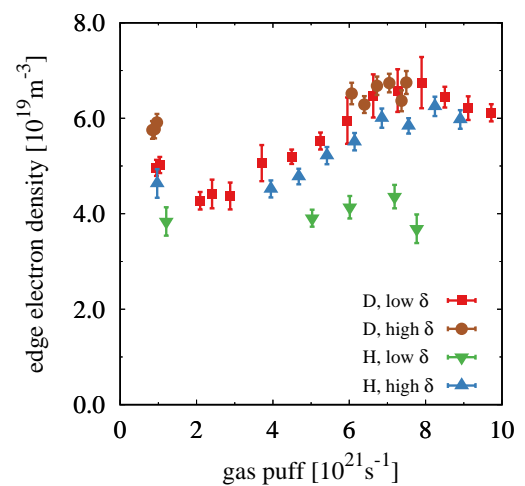

FIG. 3: Density response to gas puffing for $I_{\mathrm{p}}=0.8 \mathrm{MA}$ discharges with different triangularity and main ions. The edge density is the taken locally at $\rho_{\mathrm{pol}}=0.9$ and the gas is puffed from divertor valves. 

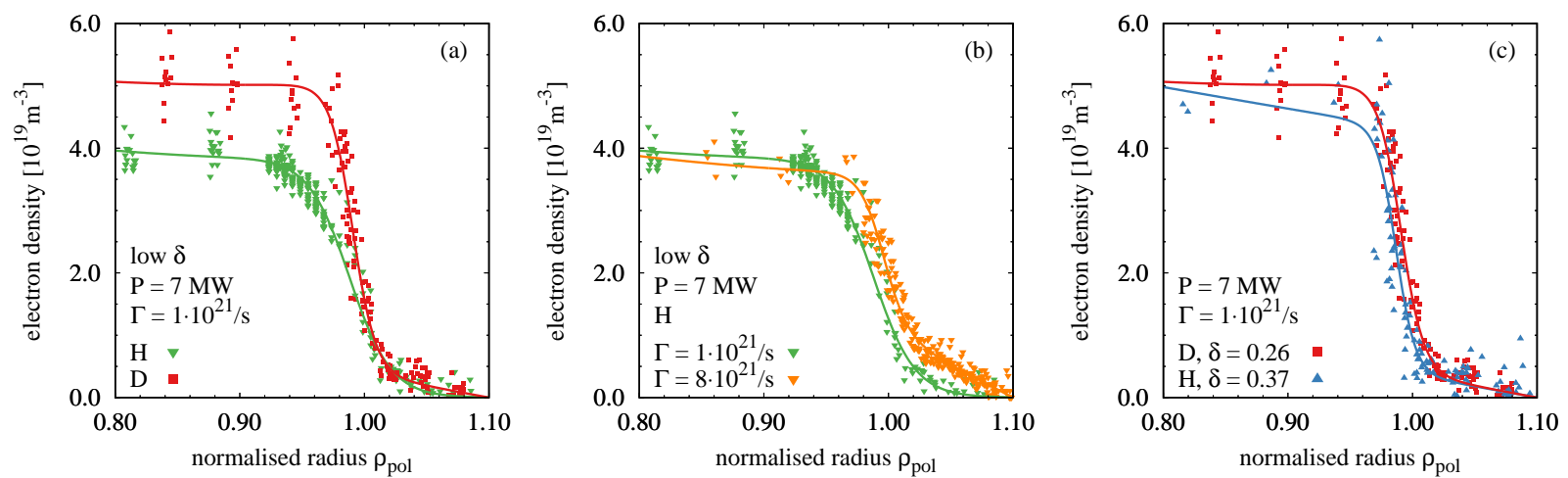

FIG. 4: Comparison of edge electron density profiles for matched engineering parameters while changing main ion mass (ID 4, 8) (a), changing gas puff levels in hydrogen (ID 4, 2) (b) and changing main ion mass along with triangularity (ID 8, 5) (c). The lines are fits to the data.

and $\mathrm{D}$ plasmas for low triangularity. However, at higher triangularity $\delta \geq 0.35$ the density in hydrogen recovers its sensitivity to the particle source and the $\mathrm{H}$ and $\mathrm{D}$ densities are again within $10 \%$ difference. The Greenwald density for these plasmas is $n_{\mathrm{GW}}=10^{20} \mathrm{~m}^{-3}$.

To illustrate the density response in more detail, figure 4 shows the pre-ELM edge density profiles. For low gas fuelling levels and matched engineering parameters (a) the $\mathrm{H}$ pedestal top density is not only lower, but the pedestal gradient is also flatter than in the D reference. In the scrape-offlayer SOL, $\rho_{\text {pol }}>1.0$, the densities are again similar, a result of the same gas puff level. This is a fairly common observation for isotope studies in H-mode as discussed in the introduction.

The pedestal response to gas fuelling in $\mathrm{H}$ plasmas with low triangularity is shown in figure 4 (b). The separatrix and SOL densities increase, however, the pedestal top density remains unaffected by the additional gas. Effectively this results in an outward shift of the density profile. This is similar to observations in deuterium, however, there the particle source needs to be considerably larger before a shift in the density is observed similar to the one in hydrogen [31].

The direct consequence is that at low triangularity we cannot achieve a match of pedestal top densities between $\mathrm{H}$ and $\mathrm{D}$ by varying the gas puff rates. The solution is shown in figure 4 (c) where in $\mathrm{H}$ the triangularity is increased while keeping the gas fuelling the same as in D. Now a match in the density pedestal - top and gradient - is achieved in $\mathrm{H}$ and $\mathrm{D}$. This behaviour is again similar to what is known for deuterium plasmas where the triangularity influences pedestal stability and result in higher densities [2,32]. Although, the impact of the triangularity on the edge density is in line with expectations, its magnitude is not understood. In particular, why its impact is stronger in hydrogen than in deuterium for higher gas puff levels. The observations suggest that it is an interplay between ELM stability, inter ELM transport and also the profile recovery after an ELM. The latter is strongly influenced by particle fluxes and could explain why differences are observed with changing gas puffing and different heating schemes.

\section{Electron temperature:}

For low gas puffing ranges the electron temperatures show similarly steep pedestals both in $\mathrm{H}$ and D. The pedestal top temperatures are also fairly similar despite different mass number, in hydrogen they are even slightly higher which is in line with the lower density. A difference is 

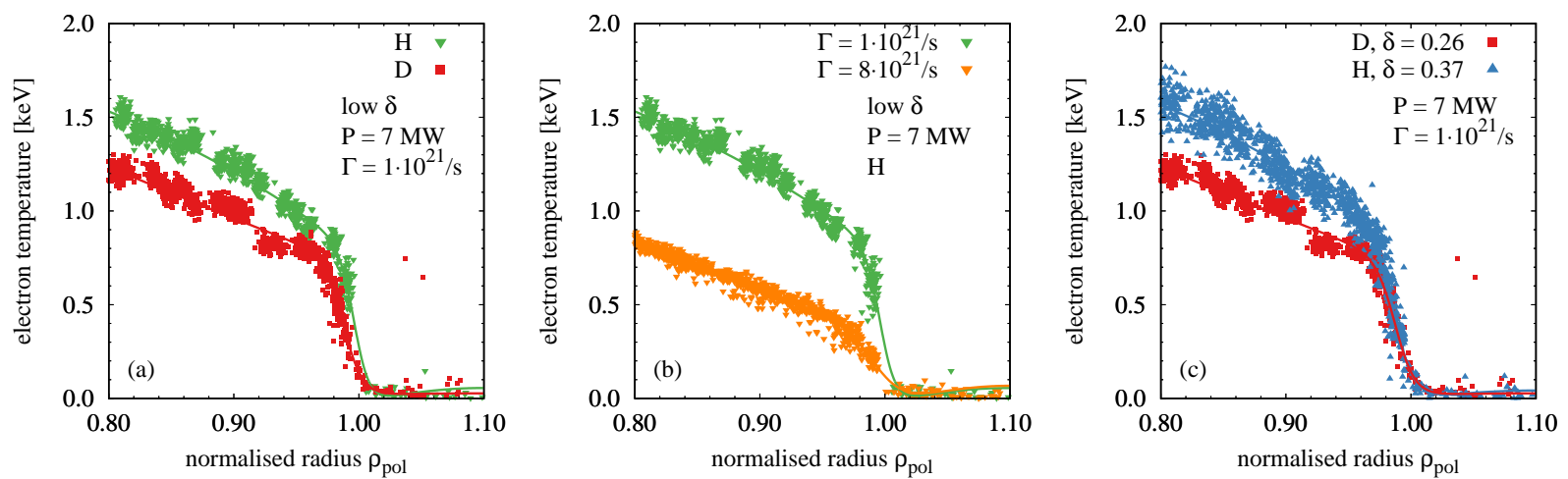

FIG. 5: Comparison of edge electron temperature profiles for matched engineering parameters while changing main ion mass (ID 4, 8) (a), changing gas puff levels in hydrogen (ID 4, 2) (b) and changing main ion mass along with triangularity (ID 8, 5) (c). The lines are fits to the data.
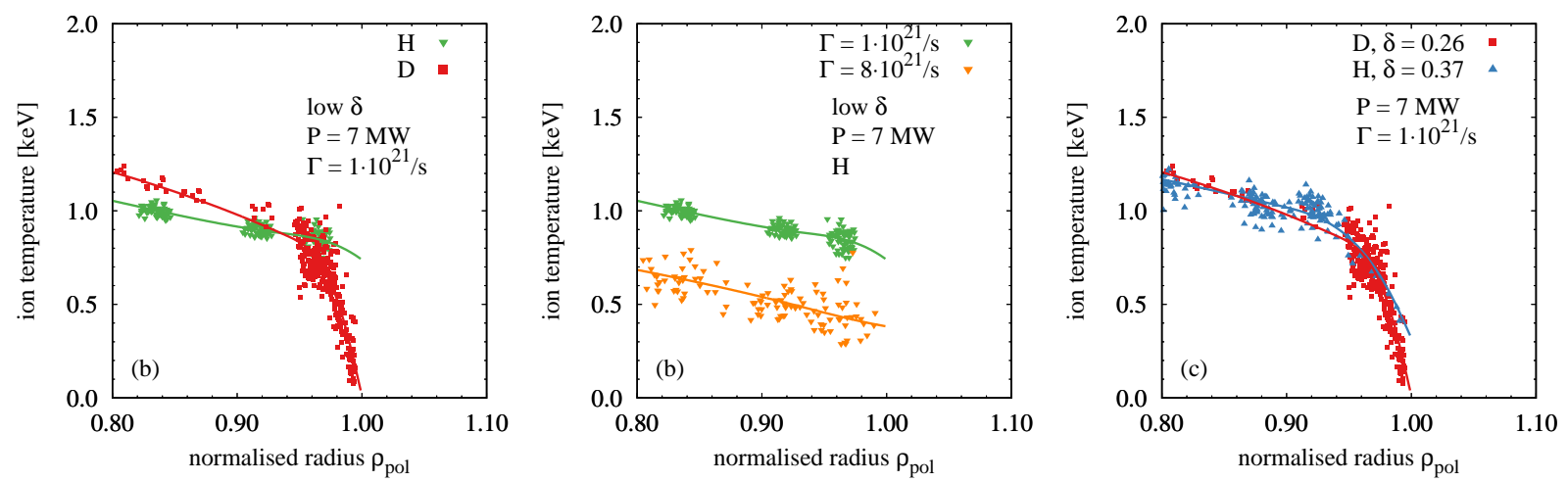

FIG. 6: Comparison of edge ion temperature profiles for matched engineering parameters while changing main ion mass (ID 4, 8) (a), changing gas puff levels in hydrogen (ID 4, 2) (b) and changing main ion mass along with triangularity (ID 8, 5) (c). The lines are fits to the data.

observed in the temperature gradient right inside of the pedestal top around $\rho_{\text {pol }} \sim 0.95$, where the hydrogen plasmas exhibit a steeper gradient as shown in figure 5 (a) and (c).

The most severe impact on the pedestal is observed in hydrogen when increasing the gas puffing in figure 5 (b). At constant heating power and constant pedestal top density the temperature drops by $50 \%$. Here the heating power is well above the L-H power threshold, $P_{\text {heat }}>2 P_{\mathrm{LH}}$. So with higher gas fuelling a dramatic loss in pedestal pressure is observed in $\mathrm{H}$, something not seen in D for similar plasma parameters. Depending on the operational regime, a strong isotopic dependence of the pedestal confinement is observed (high gas fuelling and low triangularity) or not observed (low gas fuelling and/or high triangularity). An overview of the confinement time of these discharges is given in section 2.2 .7 and figure 17

\section{Ion temperature:}

Analogous to the electron density in figure 4 and the electron temperature in figure 5 the edge ion temperature is shown in figure 6. High resolution edge measurements are not available for all discharges, but even for these cases the outermost core measurements are sufficient to draw the following conclusions. In the presence of high gas puffing at low $\delta$ in hydrogen, the ion temperature at the pedestal top in figure 6 (b) collapses in a similar way as does the electron temperature in figure (b). Inside the pedestal top, while the electron temperature gradients were consistently 

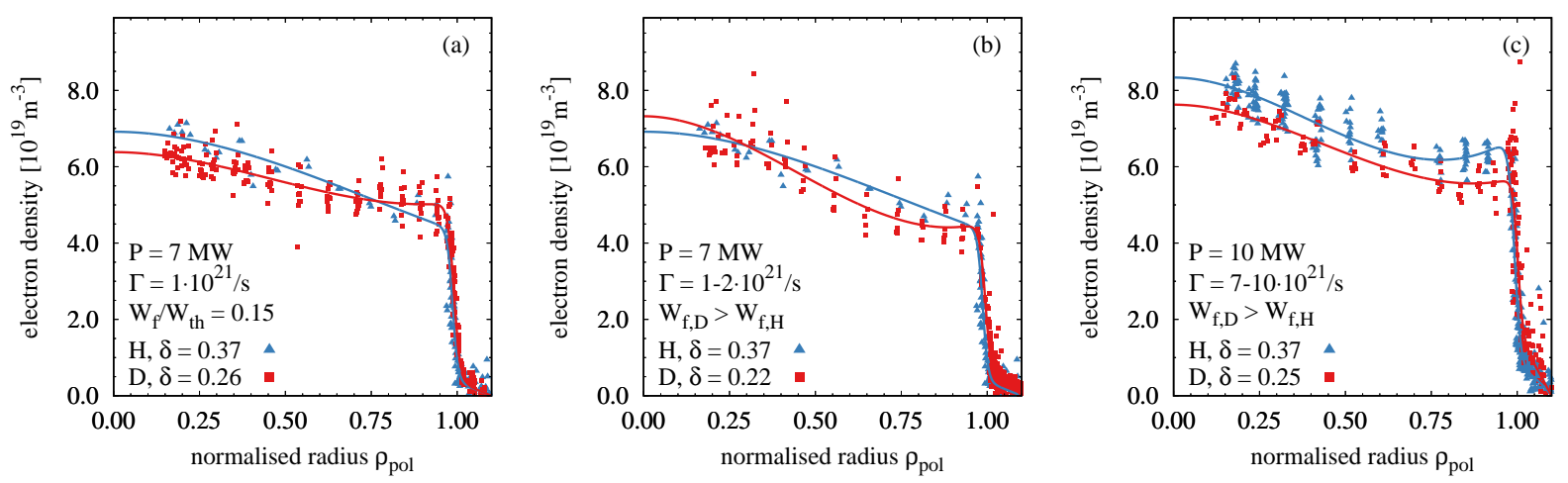

FIG. 7: Electron density profiles for $H$ and $D$ comparison discharges with matched pedestal top density. Low gas and low heating with matched $W_{\text {fast }} / W_{\mathrm{th}}(I D 5,8)$ (a), matched toroidal rotation $(I D 5,1)$ (b) and high gas and high heating (ID 3 , 7) (c).

flatter in $\mathrm{D}$ than $\mathrm{H}$, the ion temperature gradients are more similar for $\mathrm{D}$ and $\mathrm{H}$, in particular, for the comparison pair 5, 8 with different triangularity but matched pedestal top density figure 6 (c).

\subsection{Plasma core}

In section 2.1 we showed that the edge pedestal can, under certain conditions, exhibit a strong isotopic dependence. This isotopic dependence can be mitigated using higher triangularity in hydrogen, i.e. H plasmas with high $\delta$ and D plasmas with low $\delta$, but otherwise the same parameters, have similar pedestal conditions. When interested in the physics of the plasma core it is beneficial to remove edge effects experimentally. Therefore, for the analysis of the plasma core, high $\delta \mathrm{H}$ plasmas are compared with low $\delta$ D plasmas. For the core comparison we chose two different heating power levels $P_{\text {tot }}=7 \mathrm{MW}($ ID $1,5,8)$ and $P_{\text {tot }}=10 \mathrm{MW}$ (ID 3, 7). The low power level has to be well above the LH-threshold in $\mathrm{H}$, the high power level was chosen to be below core MHD onset in $\mathrm{D}$. The high heating power is also restricted to high densities due to increased shine through of $\mathrm{H}-\mathrm{NBI}$. With $\mathrm{H}-\mathrm{NBI}$ and D-NBI injection at AUG one cannot match rotation and fast-ion content simultaneously which is the reason for choosing 3 discharges at low power. There is the comparison of pair 5,8 with matched $W_{\text {fast }} / W_{\text {th }}$ but different core rotation and the pair 5, 1 with matched rotation but different $W_{\text {fast }} / W_{\text {th. }}$. These two discharge pairs and the high power pair 3, 7 are described in detail in this section.

\subsubsection{Electron density}

Figure 1 shows that with different $\delta$ the density is matched within $10 \%$ for the chosen $\mathrm{H}$ and D plasma comparison. This is true for the pedestal density as well as for the density peaking.

\subsubsection{Electron temperature}

The core temperature profiles for the discharges chosen to study core transport are shown in figure 8. The low heating power cases (a) and (b), show the differences for $\rho_{\text {pol }}>0.6$ as discussed in section 2.1, while in the core the absolute electron temperature is matched very well. For the high power, high gas comparison (c), the edge electron temperatures match well, while in the core $T_{\mathrm{e}, D}>T_{\mathrm{e}, H}$, however, the difference remains below $15 \%$. 

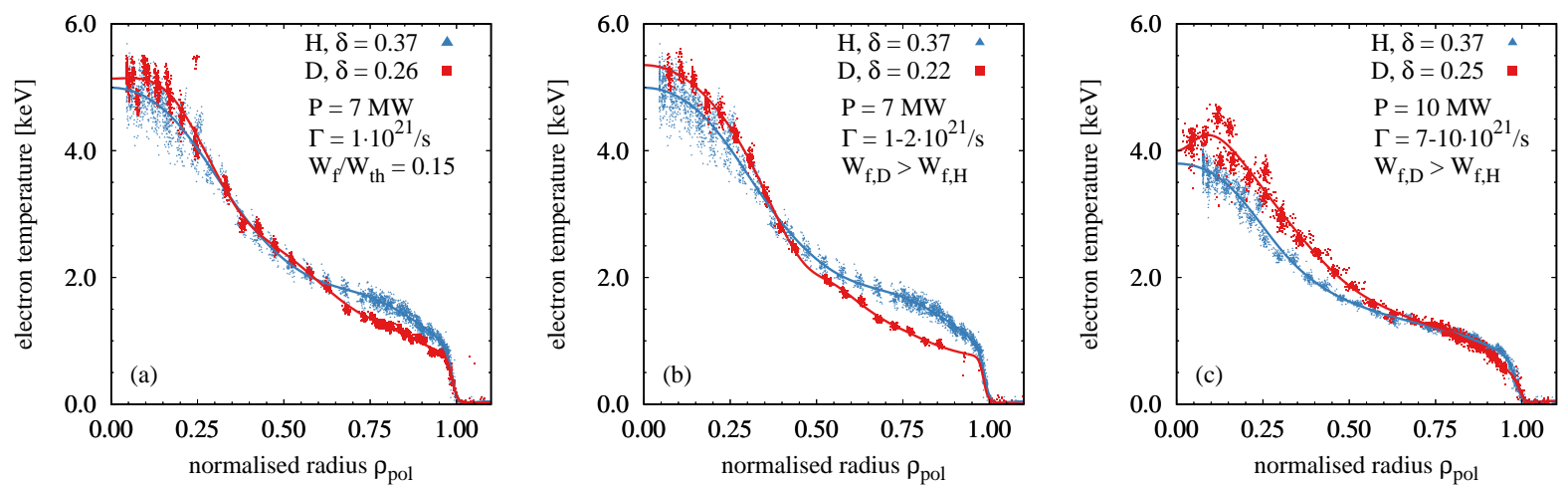

FIG. 8: Electron temperature profiles for $H$ and $D$ discharge pairs with matched pedestal top density. Low gas and low heating with matched $W_{\text {fast }} / W_{\text {th }}(I D 5,8)$ (a), matched toroidal rotation $(I D 5,1)$ (b) and high gas and high heating (ID 3, 7) (c).
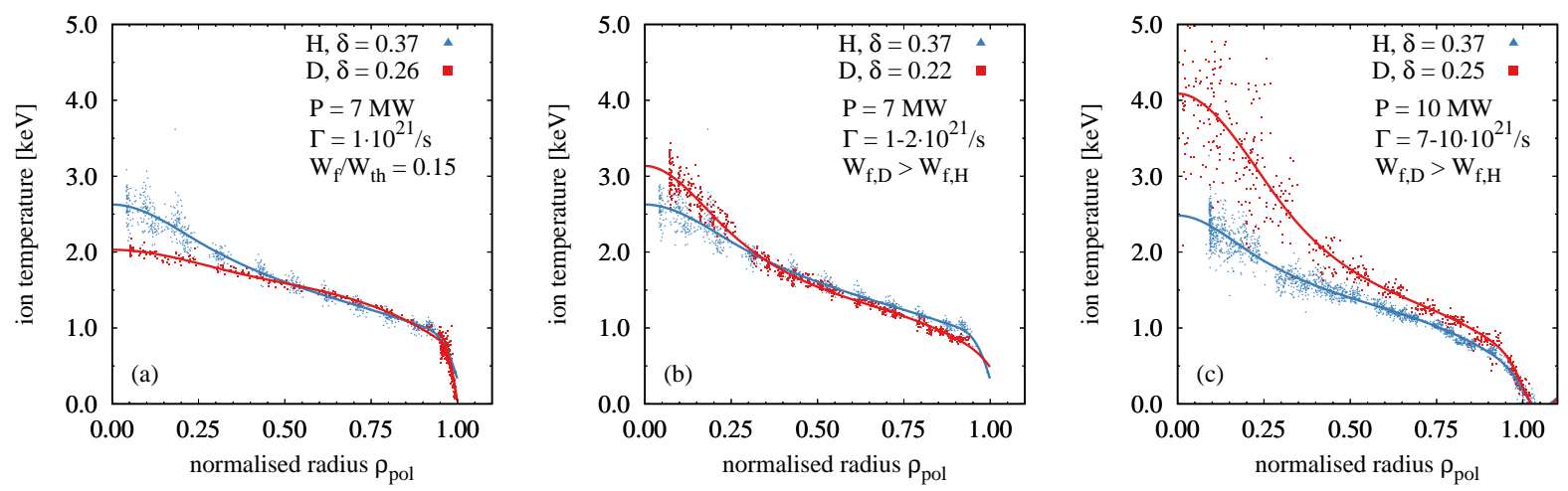

FIG. 9: Ion temperature profiles for $H$ and $D$ discharge pairs with matched pedestal top density. Low gas and low heating with matched $W_{\text {fast }} / W_{\text {th }}(I D 5,8)$ (a), matched toroidal rotation $(I D 5,1)$ (b) and high gas and high heating (ID 3, 7) (c).

\subsubsection{Ion temperature}

In figure 9 differences are observed in the ion temperature for the three $\mathrm{H}$ and $\mathrm{D}$ discharge pairs. For the low heating power comparisons $(\mathrm{a})+(\mathrm{b})$ we find $T_{\mathrm{i}}$ in $\mathrm{H}$ between $T_{\mathrm{i}}$ of the two $\mathrm{D}$ plasmas. While for most of the radius all 3 profiles agree within the uncertainties, they deviate towards the center of the plasma. The D plasma (ID 8) matching $W_{\text {fast }, \mathrm{H}}$ but having lower rotation has the lowest $T_{\mathrm{i}}$ and the $\mathrm{D}$ plasma (ID 1) matching rotation with $\mathrm{H}$ while having more fast ions has the highest $T_{\mathrm{i}}$. A more significant difference in $T_{\mathrm{i}}$ is observed for the high power case which also has higher $n_{\mathrm{e}}$ figure 9 (c) where the ion temperature is more peaked in $\mathrm{D}$ resulting in $T_{\mathrm{i}, \mathrm{D}}(0) \simeq 1.6 T_{\mathrm{i}, \mathrm{H}}(0)$. This is the only significant difference observed in the core density and temperature profiles for $\mathrm{H}$ and $\mathrm{D}$ plasmas.

\subsubsection{Rotation}

The important factors determining the rotation are momentum confinement, torque input and plasma inertia. The momentum confinement is strongly linked to the energy confinement [33]. Consequently, reduced energy confinement is accompanied by lower rotation. This is also observed in AUG and illustrated in figure 10 where rotation profiles (b) are shown for matched NBI torque input (a) but different triangularity. The discharge with higher $\delta$ (ID 5) and improved edge energy confinement is rotating faster than its counterpart (ID 4), despite having also higher density. This 

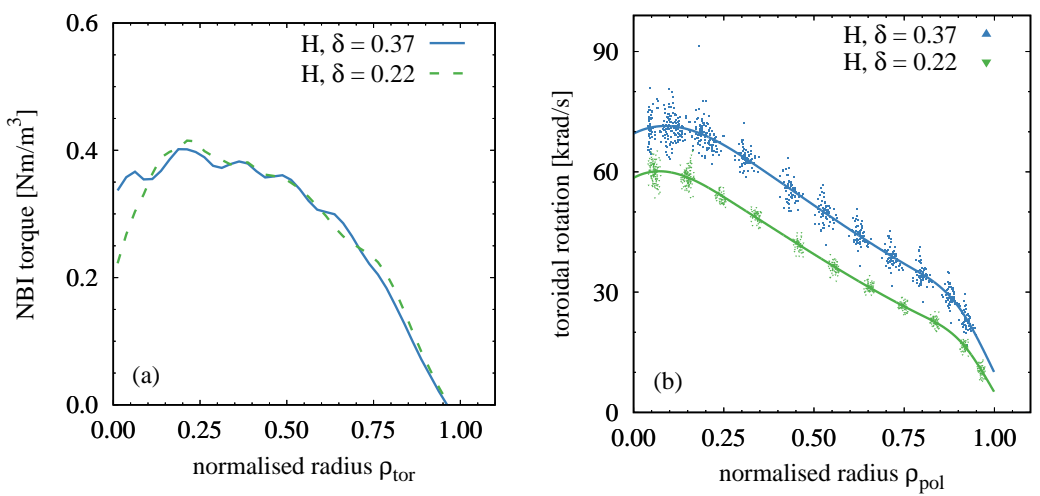

FIG. 10: Discharges with the same NBI torque input (a) but different triangularity show different plasma rotation (b) (ID 4, 5).
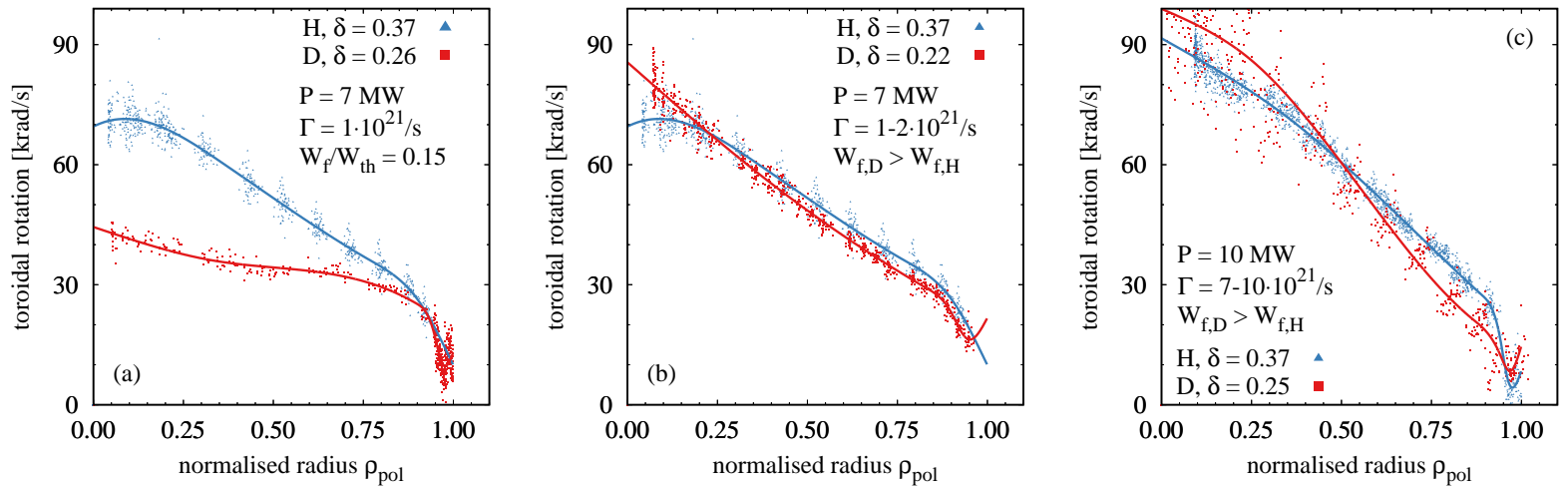

FIG. 11: Toroidal rotation profiles for $H$ and $D$ discharge pairs with matched pedestal top density. Low gas and low heating with matched $W_{\text {fast }} / W_{\mathrm{th}}(I D 5,8)$ (a), matched toroidal rotation $(I D 5,1)$ (b) and high gas and high heating (ID 3, 7) (c).

highlights the importance of matched pedestal confinement to achieve similar rotation velocities. Typically, H plasmas are heated with H-NBI and D plasmas with D-NBI, therefore, the torque input will be different. For similar heating power at nominal NBI voltages the total torque induced by D-NBI will be 1.2-1.6 of that by H-NBI, depending on the geometry of the utilized NBI sources. At the same time the inertia of a hydrogen plasma is lower compensating for the difference in torque.

The rotation profiles for the set of comparison discharges are shown in figure11. At nominal beam voltage for $\mathrm{H}$ and $\mathrm{D}$ the plasmas rotate similarly fast $(\mathrm{b})+(\mathrm{c})$. In the case (a) where the D-NBI voltage was reduced to match the fast-ion content of H-NBI, the core rotation drops significantly as the beam deposition shifts towards the edge of the plasma.

\subsubsection{Fast-ions}

The fast-ion content in hydrogen and deuterium is naturally quite different. The reason for this is the difference in the classical fast-ion slowing down time 34]

$$
\tau=\frac{t_{\mathrm{s}}}{3} \ln \left(1+\left(\frac{W}{W_{\text {crit }}}\right)^{3 / 2}\right)
$$

where $t_{\mathrm{s}}$ is the Spitzer time and $W$ is the neutral beam energy which for the two NBI systems on AUG is larger in deuterium with $W_{\mathrm{D}}=93 \mathrm{keV}, 60 \mathrm{keV}$ than in $\mathrm{H}$ with $W_{\mathrm{H}}=72 \mathrm{keV}, 55 \mathrm{keV}$. $W_{\text {crit }}$ 

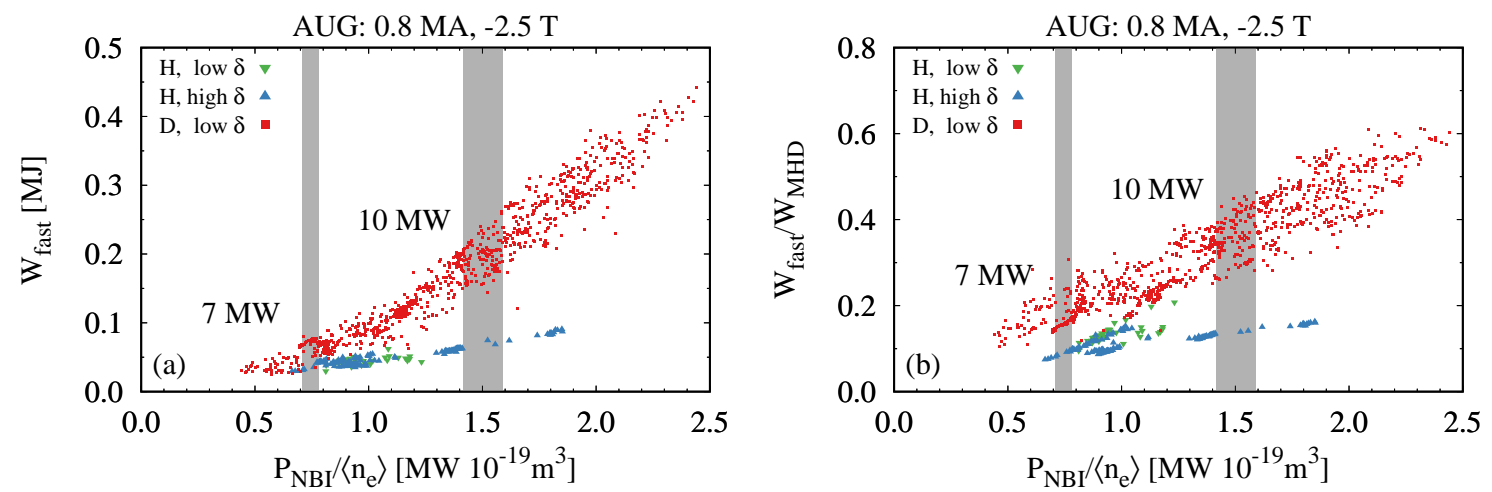

FIG. 12: RABBIT calculations of the fast-ion energy (a) and normalized fast-ion energy (b) plotted against the NBI heating power per density for a data base of $0.8 \mathrm{MA}$ discharges. The parameter range for the dedicated discharges listed in Table $\square$ is highlighted by the grey shaded area.
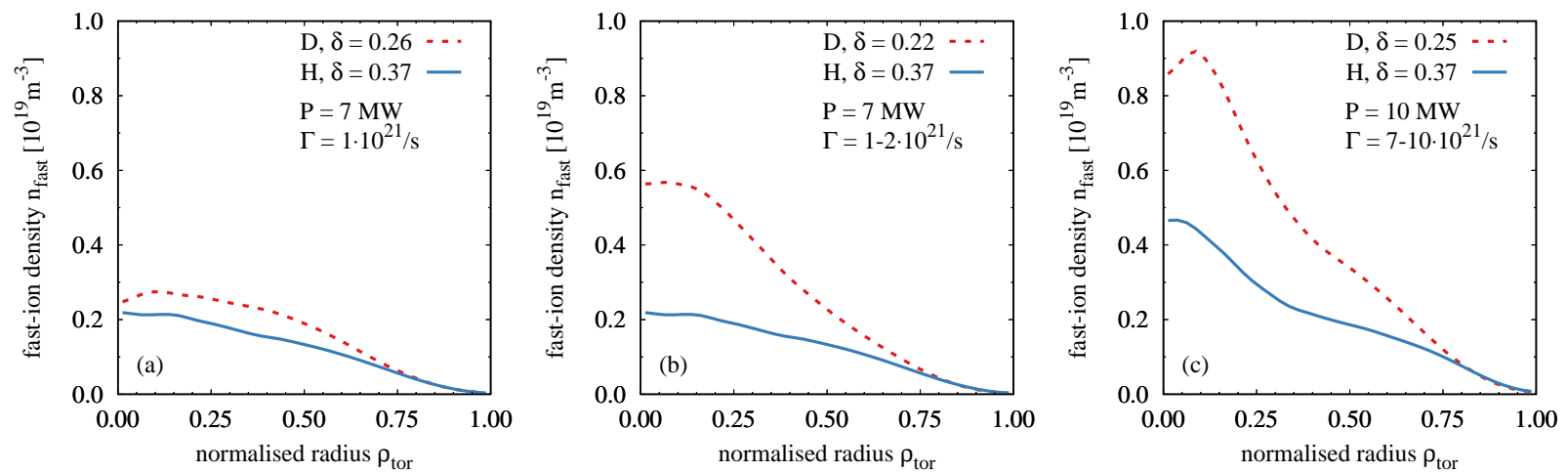

FIG. 13: Fast-ion density and pressure calculated with NUBEAM for low heating with varying rotation (a) (ID 8, 5) and matched rotation (b) (ID 1,5) and high heating (c) (ID 3, 7$)$. 
is the critical energy denoting the transition between slowing down to electrons vs. ions and for pure plasmas $W_{\text {crit }} \propto A^{1 / 3}$. For the AUG beam setup this means the mass dependence of $W / W_{\text {crit }}$ mostly cancels, in consequence the isotope dependence of $t_{\mathrm{s}}$ is relevant for the overall behaviour

$$
t_{\mathrm{s}} \propto \frac{A T_{\mathrm{e}}^{3 / 2}}{Z^{2} n_{\mathrm{e}} \ln \Lambda} \propto A .
$$

One should note that this simplification is only valid for pure plasmas - i.e. H-NBI into H or D-NBI into D - and not for mixtures. From the classical slowing down time a significant difference in fast-ion content is expected for different main ion masses even for similar background plasma parameters. The RABBIT [35] code is used to calculate the classically expected fast-ion energy for a large data set of AUG discharges. This calculation does not include non-classical influences from e.g. mode activity (like Alfven modes or neoclassical tearing modes (NTM)), these can occur at high fast-ion content and/or high $\beta_{\mathrm{N}}$. As a result the calculated values might be overestimated, since the database was not screened for mode activity. However, the relative difference between $\mathrm{H}$ and D will be similar. Note the individual discharges discussed throughout this paper do not have an NTM present, neither in H nor in D. In figure 12 (a) the fast-ion energy $W_{\text {fast }}$, as calculated by RABBIT, is plotted against the fast-ion input per density $P_{\mathrm{NBI}} /\left\langle n_{\mathrm{e}}\right\rangle$ for the AUG database and illustrates the different fast-ion energies with the main ion mass. The relative fast-ion energy $W_{\text {fast }} / W_{\text {MHD }}$ is plotted in figure 12 (b) and shows the increasing importance of fast ions on the total stored energy for increasing heating power per particle. This behaviour is expected because with higher heating power more fast ions are introduced into the plasma and the resulting higher temperature results in longer slowing down, so $W_{\text {fast }} \propto P_{\mathrm{NBI}}^{\alpha}$ with $\alpha>1$ - here $\alpha=1.67$. Higher thermal temperatures generally result in higher heat transport, i.e. $W_{\text {th }} \propto P_{\text {heat }}^{\beta}$ with $\beta<1$, with e.g. $\beta=0.31$ as in the ITER Physics Basis IPB98(y,2) scaling [36. Figure 12 (b) also illustrates that for H-NBI into $\mathrm{H}$ discharges the fast ions amount to only $20 \%$ of the total energy, while in D this value can go up to $50 \%$. This general database view is reproduced by the NUBEAM [37] module of the TRAnsP [38] simulations for the discharges presented here. Radial profiles of the fast-ion density $n_{\text {fast }}$ are illustrated in figure 13 and highlight the differences between these discharges. The match of fast-ion content between $\mathrm{H}$ and $\mathrm{D}$ in figure 13 (a) is achieved by reducing the D-NBI injection voltage. For the fast-ion pressure $p_{\text {fast }}$ the differences between H and D will slightly increase due to higher fast-ion temperatures in D for (b) and (c).

Diagnostic measurements of the fast ions with the FIDA (fast-ion $D_{\alpha}$ ) diagnostic also support the trend described above. Radial profiles of the FIDA radiation, integrated over certain wavelength ranges - corresponding to different fast-ion energies - are shown in figure 14, The FIDA radiation is shown normalised over the beam emission (BES) intensity, allowing for the neutral beam attenuation to be taken into account, a method described in detail in [39]. As such, it can be used as a proxy for the fast-ion population in the plasma. While the FIDA measurements are sampling only a part of the pitch-velocity space, the measurements support the above analysis indicating a larger fast-ion population in the core of the D plasma compared to the $\mathrm{H}$ plasma. Unfortunately, the FIDA edge channels do not have the time resolution to resolve ELMs and therefore, the measurements are corrupted by ELMs [40] and no clear conclusions can be drawn for the edge. 


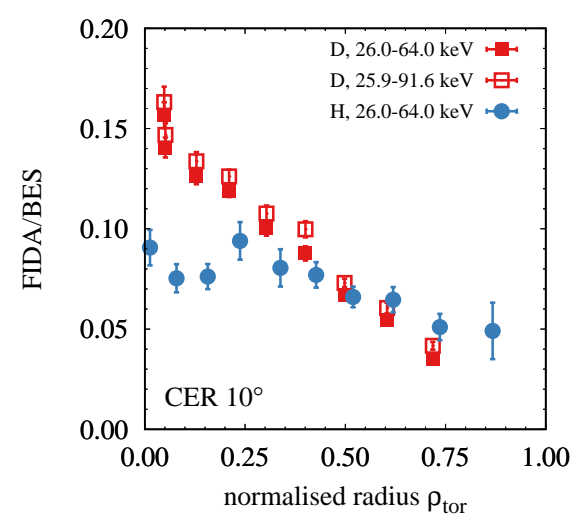

FIG. 14: FIDA intensity normalised to the beam emission signal (BES) used as proxy for the fast-ion density. The data points are from discharge pair 3, 7.

\subsubsection{Power balance}

In the previous sections we discussed temperature and density profiles, apart from $T_{\mathrm{i}}$ for the high heating case, the profiles matched fairly well for the selected comparison discharges. While the experiments were optimised to also match the heating profiles, small differences could not completely be avoided. To consider these differences we perform a power balance analysis and compare the heat diffusivity $\chi$ for the different discharges and heat channels. The auxiliary and Ohmic heating profiles are calculated with TRANSP and the radiation profiles are a deconvolution of bolometer measurements. The uncertainties for $\chi$ are determined taking into account the uncertainty of the density, temperature gradients and the electron-ion equipartition, the input power is assumed to be without error. The comparison of the diffusivities will illustrate the differences in transport properties and allows us to draw conclusions on potential isotopic dependencies.

For low fast-ion content and matched rotation profiles (ID 5, 1) the ion heat diffusivity is the same for $\mathrm{H}$ and $\mathrm{D}$ within uncertainties as shown in figure 15 (e). This results in matched temperature profiles shown in figure 9 (b). When trying to match the fast-ion pressure and thereby relaxing the match of rotation profiles (ID 5,8) the plasma with the flatter rotation profile - here the D plasma - shows larger diffusivities in the core of the plasma, seen in figure 15(d). The third pair of discharges - the one at high heating power (ID 3, 7) - has similar rotation profiles, but different fast-ion pressure and more importantly a high $W_{\text {fast }} / W_{\text {th }}=0.37$ in $\mathrm{D}$. This case, shown in figure 15 (f), has an ion heat diffusivity lower by a factor of $\sim 2$ for $\rho_{\text {tor }}<0.4\left(\approx \rho_{\text {pol }}<0.55\right)$ in D (where $\rho_{\text {tor }}=\sqrt{\Phi / \Phi_{\max }}, \Phi$ being the toroidal magnetic flux). This coincides with a higher $T_{\mathrm{i}}$ and stronger $T_{\mathrm{i}}$ peaking in the core of the D plasma. The electron heat diffusivities figure 15 (a, b, c) follow largely the trends found for the ion heat transport channel with one striking exception. Inside of the pedestal top $\chi_{\mathrm{e}}$ is found higher for $\mathrm{D}$ plasmas consistently over all pairs. This is also reflected in the temperature profiles shown in figure 8 . This could be a result of the different triangularity, then $\delta$ would have an isotope dependent impact on the electron heat transport, similar to the observation made for the density. As shown in figure 16 varying the shape results in different $\chi_{\mathrm{e}}$ at the edge of $\mathrm{H}$ plasmas (a) while it has little impact for the edge of a D plasma (b). The ion temperatures lack of good measurements in the pedestal region for cases 5 and 8 , so no 

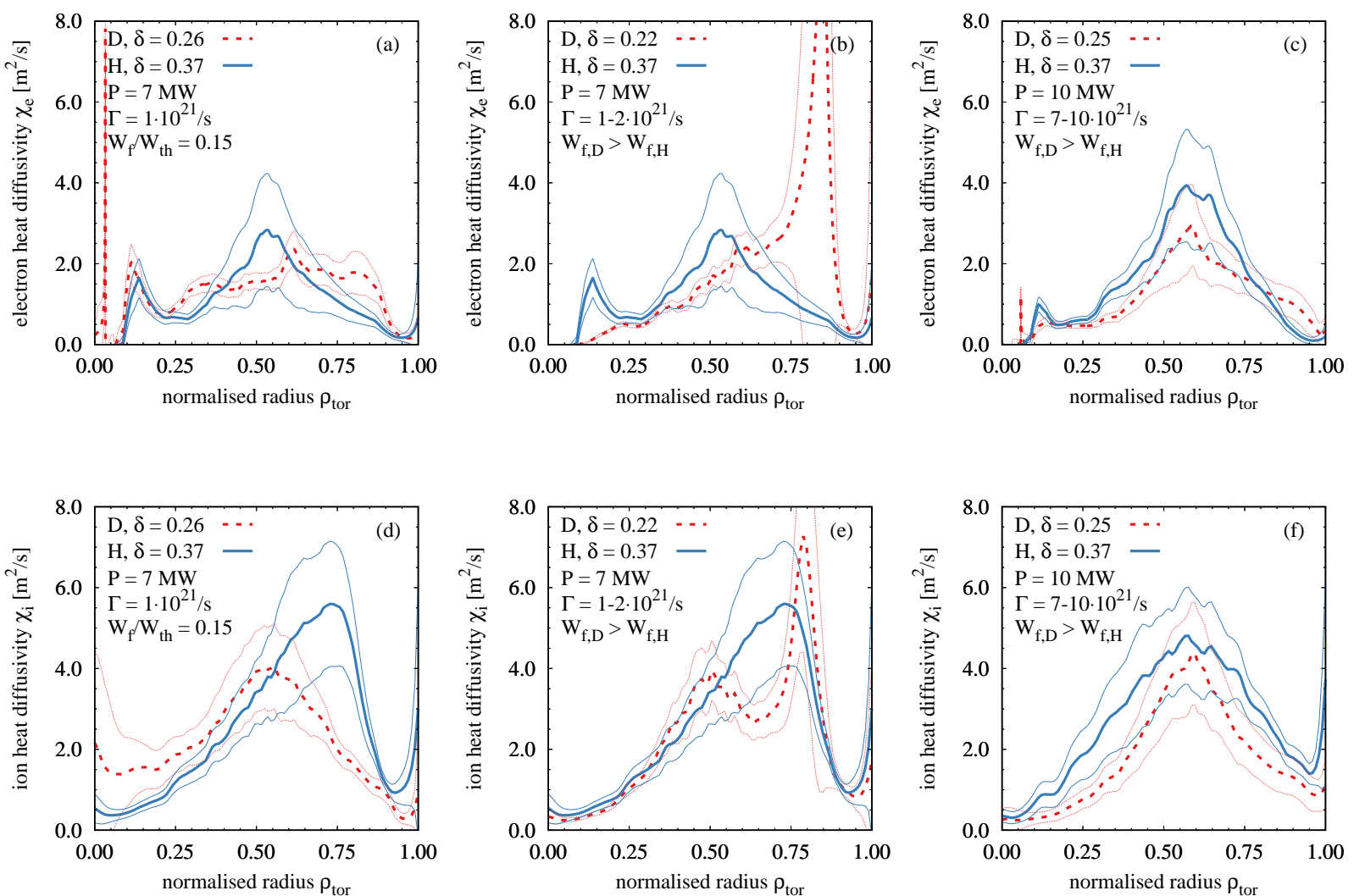

FIG. 15: Electron and ion heat diffusivities for the $H$ and $D$ comparison for the discharges with matched pedestal top density. Low gas and low heating with different rotation (ID 5, 8) (a, d), matched rotation (ID 5, 1) (b, e) and high gas and high heating with different $W_{\text {fast }}(I D$ 3, 7) $(c, f)$. The thin lines represent the uncertainties of the diffusivities (thick lines).

robust conclusions can be deduced for the ion channel at the plasma edge. In the core $\rho_{\text {tor }}<0.5$ $\left(\approx \rho_{\text {pol }}<0.7\right)$ the impact of the different triangularity is less significant for either isotope.

\subsubsection{Overview}

To give an overview of the different parameter changes discussed in the previous sections and their impact, we compare ratios of confinement times for the discharges with $7 \mathrm{MW}$. This is illustrated in figure 17 where on the $\mathrm{x}$-axis stepwise either gas puff or triangularity or isotope mass is varied from left to right and on the y-axis the confinement time is plotted normalized to the hydrogen plasma with low gas puff and high triangularity at moderate heating power (ID 5). The largest impact on the confinement time $\tau_{\mathrm{E}}$ is observed in hydrogen. Increasing the gas puffing for low $\delta$ (ID 2,4) can reduce the confinement time by $30 \%$, while a similar increase in gas puff for high $\delta$ plasmas (ID 5,6) will result in a negligible $4 \%$ decrease of $\tau_{\mathrm{E}}$. Increasing $\delta$ at low gas puffing (ID 4,5 ) will increase $\tau_{\mathrm{E}}$ by $25 \%$, consequently increasing $\delta$ at high gas puffing (ID 2,6) results in $70 \%$ higher confinement time. This is all for the same heating power and in hydrogen plasmas. The main driver for these differences appears to be located in the edge pedestal. The D cases show only little variation in confinement time when varying gas and triangularity. When comparing the low $\delta$, low gas puff case between $\mathrm{H}$ and D (ID 1,4) we find the IPB98(y,2) mass dependence $\tau_{\mathrm{E}}^{\mathrm{D}} / \tau_{\mathrm{E}}^{\mathrm{H}}=1.15 \sim 2^{0.19}$.

For discharges with higher power levels we utilize the data set introduced in section 2.2 .5 which consists of all $800 \mathrm{kA}$ and $-2.5 \mathrm{~T}$ discharges (consistent with other parts of the paper) from 


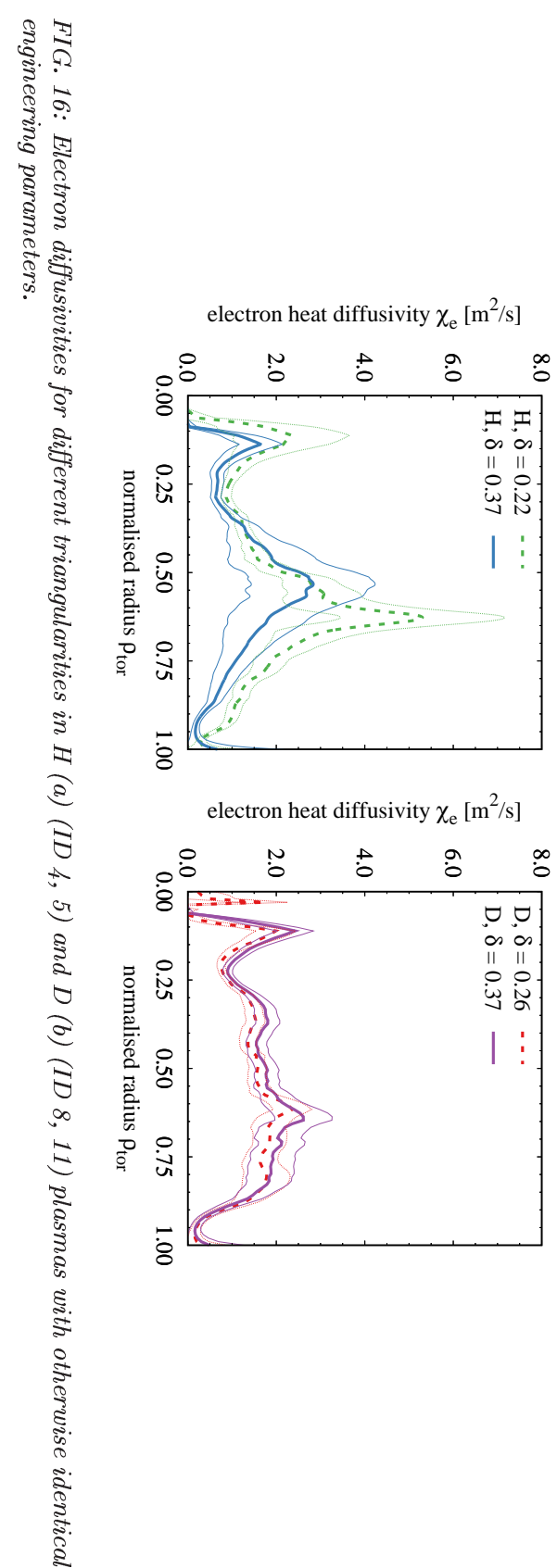




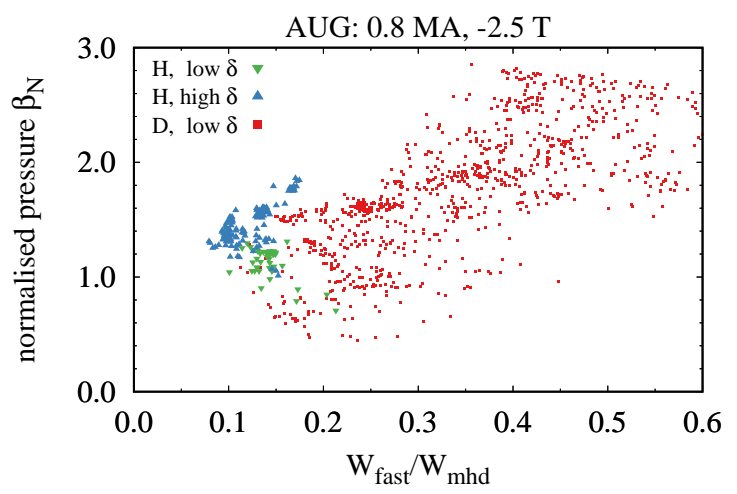

FIG. 18: Normalized global pressure $\beta_{\mathrm{N}}$ plotted against the fast-ion energy fraction determined with the RABBIT code.

the 2016-2018 AUG campaigns with the selection criteria of $P_{\mathrm{NBI}}>2 \mathrm{MW}, P_{\mathrm{rad}} / P_{\text {heat }}<0.4$, $f_{\mathrm{ELM}}<150 \mathrm{~Hz}$ and $\delta<0.3$ for $\mathrm{D}$ and $f_{\mathrm{ELM}}<120 \mathrm{~Hz}$ for $\mathrm{H}$. This essentially restricts the data set to type-I ELMy H-modes with NBI heating which do not suffer from impurity accumulation. It also excludes $\mathrm{H}$ discharges with high gas puffing and low confinement as discussed in the previous paragraph, because those exhibit higher ELM frequencies.

In figure 18 the normalized global pressure $\beta_{\mathrm{N}}$ is plotted against the fast-ion energy fraction. While the spread in the data is considerable due to different densities and heating mixes, the data illustrates that at low fast-ion content no high pressures are achieved, neither for $\mathrm{H}$ nor for $\mathrm{D}$. Larger $\beta_{\mathrm{N}}$ values are only accessible when $W_{\text {fast }} / W_{\text {mhd }}>0.25$ or $W_{\text {fast }} / W_{\text {th }}>1 / 3$.

If only the $\mathrm{D}$ data was present one could argue that the correlation of heating power and fast-ion content is a trivial explanation for this trend. However, as shown in figure 12 the correlation of heating power and fast-ion content is broken with different isotope mass. So further investigation of the influence of fast ions is warranted. Known effects of the fast ions are the main ion dilution in the core [15] and non-linear turbulence stabilisation [12,41,43] - the latter being discussed in detail in section 3.2. Both core mechanisms would result in reduced profile stiffness allowing the higher pressures which are inaccessible with the low fast-ion content in $\mathrm{H}$. With higher fast-ion content in AUG typically the ratio $P_{\mathrm{NBI}} / P_{\mathrm{ECRH}}$ increases and consequently the ion heating increases which can be beneficial for confinement [14].

\section{Modelling}

To understand the observations made in the plasma core with known physics concepts a series of simulations has been performed. To estimate the impact due to mass, fast ions, rotation and the boundary conditions predictive ASTRA simulations are performed using the quasilinear TGLF model. To better quantify the impact of different fast-ion content additional linear and non-linear local gyrokinetic simulations are done with GENE for the high power comparison.

\subsection{Astra / TGLF}

In figure 19 an overview of the ASTRA [19,20] simulations with the TGLF [17 transport model is given. The simulations are made with a recent release of TGLF and saturation rule Sat1geo [18] 

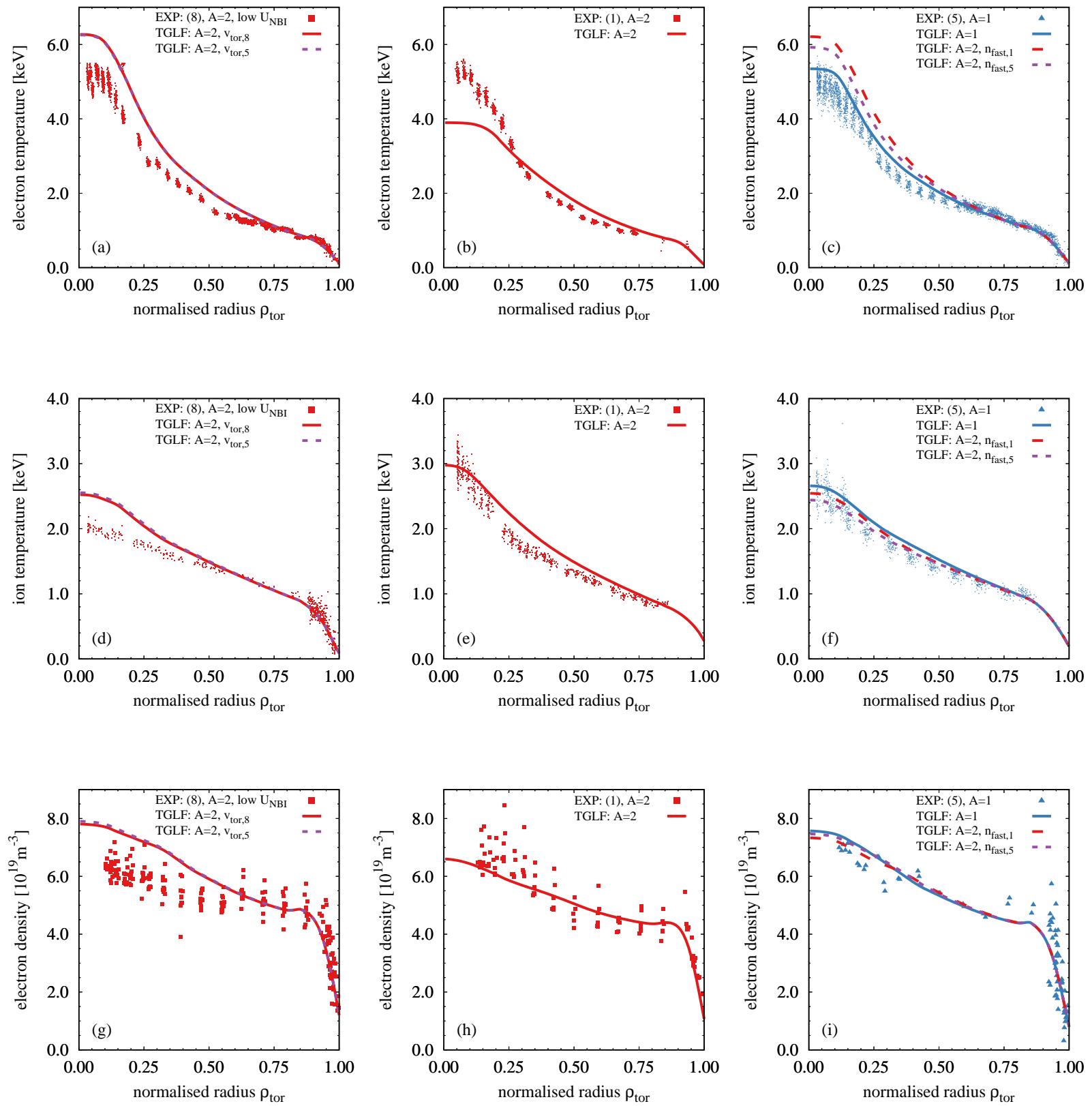

FIG. 19: TGLF predictions plotted with lines for the low heating power cases $8(D)(a, d, g), 1(D)(b, e, h)$ and $5(H)(c, f, i)$. Additional conditions are reported in the legends. The measurements for $T_{e}, T_{i}$ and $n_{e}$ are plotted with points. 
and use the experimental rotation and the TrAnsp fast-ion density and heat flux profiles. These profiles are fixed inputs and will only be varied when explicitly stated. Boundary conditions are taken from experimental measurements inside the pedestal top, at $\rho_{\text {tor }}=0.85$. The fast ions are treated as a non-thermal impurity species [12], i.e. they are removed from TGLF effectively reducing the thermal ion density $n_{\mathrm{i}}$, but no model for non-linear turbulence stabilisation is considered. Electron and ion temperatures, electron density and current density are modelled.

Low heating power: The main observation is similar to that of the experimental power balance analysis. The quality of the transport prediction can vary already without changing the isotope mass. The two D cases with different fast-ion content and rotation (ID 1,8) are somewhat over predicted by the model while the prediction for the $\mathrm{H}$ case (ID 5) matches the measurements very well. Different parameter variations are performed to identify the dominating physics in these discharges.

In figure 19 ( $\mathrm{a}, \mathrm{d}, \mathrm{g})$ we show simulations with different rotation profiles while all other parameters are fixed. The respective rotation profiles were shown figure 11 (a). Although, the rotation and its gradients are significantly different, they do not influence the transport under these conditions. The impact of the mass number $A$ is tested with the parameters of the $\mathrm{H}$ plasma and using $A=2$. The result is shown in figure 19 (c, f, i), and it does not exhibit a gyro-Bohm like mass dependence with $\chi^{\mathrm{D}}=\sqrt{2} \chi^{\mathrm{H}}$ and the main differences are due to a different heat distribution caused by the mass dependence in the equipartition. With $A=2$ the equipartition between the electron and ion heat channels is reduced and because $T_{\mathrm{e}}>T_{\mathrm{i}}$ more heat remains in the electron channel causing higher $T_{\mathrm{e}}$ while $T_{\mathrm{i}}$ is reduced. However, since electron and ion heat transport are not equal this results in a change of global confinement. This is similar to electron heated L-mode plasmas [4] where the $\mathrm{H}$ plasma had lower confinement because enhanced equipartition with $A=1$ resulted in more energy being transferred into the ion channel which exhibited higher heat transport.

Figure 19 (c, f, i) shows the results of changing the fast-ion content from $W_{\text {fast }, \mathrm{H}} / W_{\text {th }}=0.10$ to $W_{\text {fast,D }} / W_{\mathrm{th}}=0.27$. On this level of fast ions the quasilinear model predicts a negligible impact on the profiles.

The match of modelled and experimental profiles is worse for the two D cases. In the low rotation, low $W_{\text {fast }}$ case - achieved by reduced NBI voltage - transport is under predicted which results in stronger profile peaking for all channels as illustrated in figure 19 (a, d, g). In the case with the nominal NBI voltage shown in figure $19(\mathrm{~b}, \mathrm{e}, \mathrm{h})$ the profile peaking of $T_{\mathrm{i}}$ is modelled more accurately. However, the electron temperature $T_{\mathrm{e}}$ is significantly too low in the core. Uncertainties in the heat distribution, mainly due to radiation measurements profiles could be ruled out as reason for this discrepancy. The modelled $n_{\mathrm{e}}$ peaking is too high in case (ID 8) and too low in case (ID 1) as shown in figure 19 (g) and (h). This is likely a direct consequence of the modelled electron temperature which has a strong impact on the particle transport [44].

Extending this study would yield more robust insight on this deviations, however, it would not impact the main conclusion regarding the isotopic dependencies found with the quasilinear TGLF model. Namely, when the boundary conditions inside of the pedestal are matched for plasmas with different isotopes and relatively low fast-ion content, no systematic deviation due to the main 

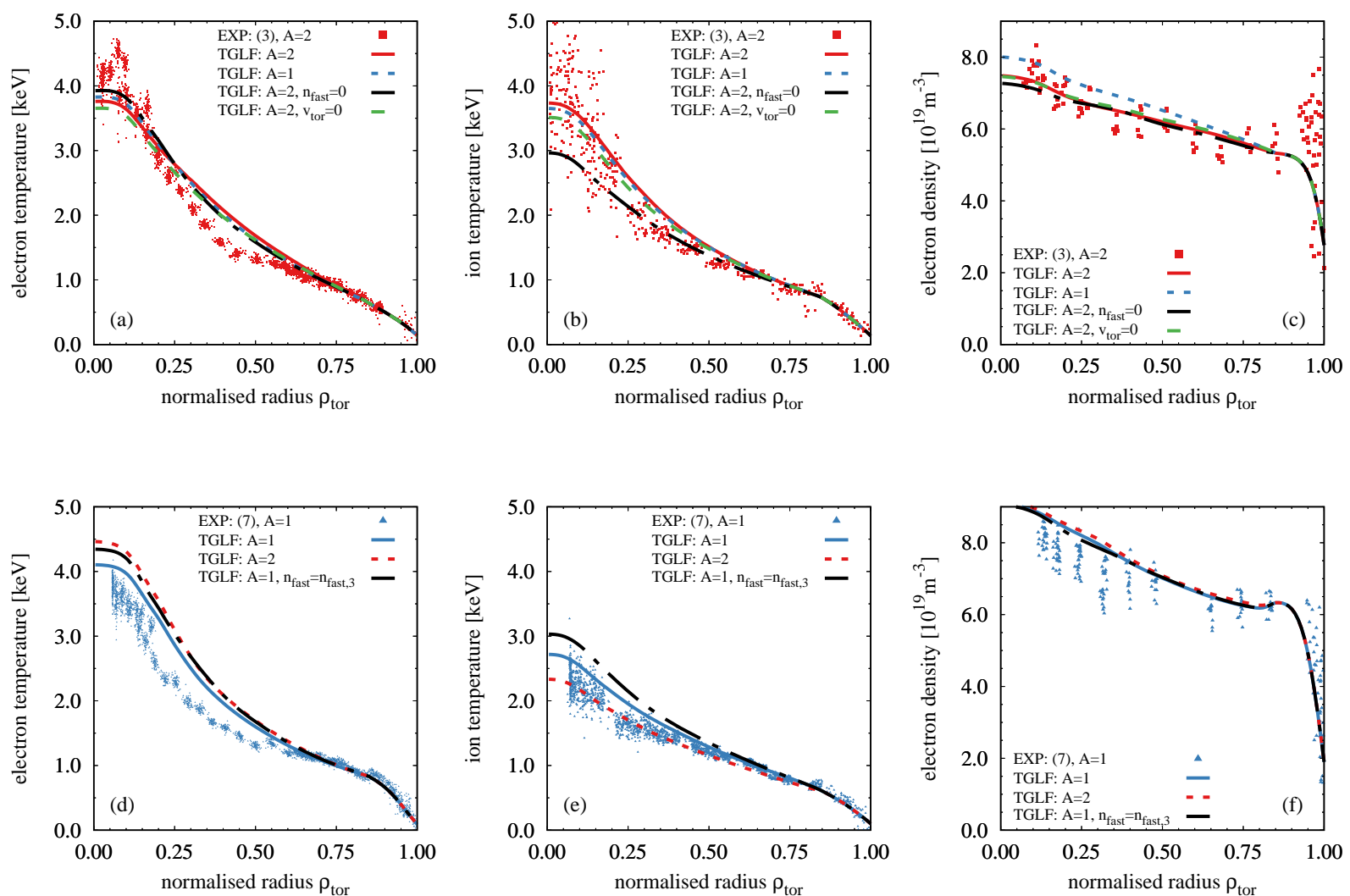

FIG. 20: TGLF predictions plotted with lines for the high heating power cases $3(D)(a, b, c)$, and 7 (H) (d, e, f). Additional conditions are reported in the legends. The measurements for $T_{e}, T_{i}$ and $n_{e}$ are plotted with points.

ion mass is observed between the experiment and TGLF predictions for $n_{\mathrm{e}}, T_{\mathrm{e}}$ and $T_{\mathrm{i}}$.

High heating power: For the high power cases $3(\mathrm{D})$ and $7(\mathrm{H})$, we test the impact of isotope mass, toroidal rotation and fast-ion density on the TGLF predictions. As illustrated in figure 20 the code predictions are in general consistent with the experimental data, in particular, the lower $T_{\mathrm{i}}$ in $\mathrm{H}$ is reproduced.

As found in the low power cases, a change of the main ion mass manifests in the temperature profiles mostly through the electron ion equipartition. In the $\mathrm{D}$ case $3, T_{\mathrm{i}} \sim T_{\mathrm{e}}$, so the energy transfer between electron and ion channel is low and the temperature profiles show only minor variations in figure 20 (a) and (b) (blue and red lines). For the $\mathrm{H}$ case 7, $T_{\mathrm{i}}<T_{\mathrm{e}}$, and, when reducing the electron to ion heat transfer by changing from $A=1$ to $A=2$, the ion temperature drops while the electrons get hotter as illustrated in figure 20 (d) and (e) (blue and red lines). The density peaking for $A=1$ is higher than in all $A=2$ cases as shown in figure 20 (c), this was observed before in AUG, where it was attributed to the reduced collisional term of the trapped particle component in the turbulent convection [45. When changing the mass number in the hydrogen case (figure 20 (c)) this effect likely cancels with the contribution due to different electron temperature peaking as discussed in section 3.1.

The toroidal rotation again plays a negligible role as shown in figure 20 (a)-(c) (green dashed). Completely removing its contribution to $E_{\mathrm{r}}$ by setting $v_{\text {tor }}=0$ reduces the central temperatures by only about $5 \%$. This means $\gamma_{E \times B}$ does play a minor role under these conditions, which 

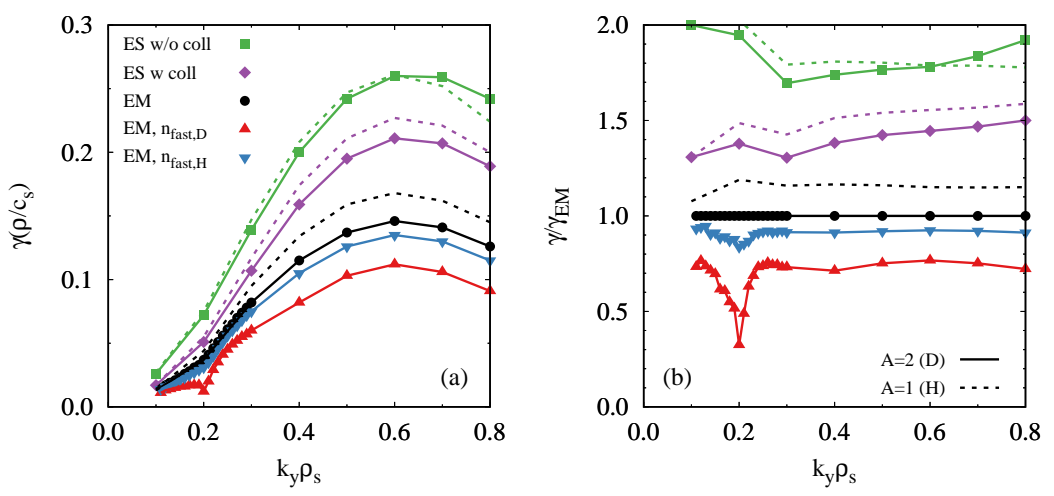

FIG. 21: Linear growth rates (a) calculated with GENE for $\rho_{\mathrm{tor}}=0.30\left(\rho_{\mathrm{pol}}=0.43\right)$ of ID 3. Growth rates normalized to the EM ones with $A=2$ (b). Solid lines correspond to the $D$ case with $A=2$, dashed lines are obtained with $A=1$.

was confirmed experimentally for comparable plasma parameters in AUG [46]. However, this is different in plasmas with internal transport barriers ITB where $\gamma_{E \times B}$ is found to have a strong impact also in the core [47,48].

In contrast to the low power cases, the fast-ion density plays a crucial role at the higher heating powers. In section 2.2.5 a significant impact of heating power and main ion mass on the fast-ion density is described. The fast-ion density is highest in the high power D plasma (ID 3) and setting $n_{\text {fast }}=0$ will result in a $20 \%$ drop of the central ion temperature as shown in figure 20 (b) (black broken line). Consequently, increasing $n_{\text {fast }}$ in the simulation of the $\mathrm{H}$ plasma (ID 7) to the level of the D plasma $n_{\text {fast }, 3}$ the $T_{\mathrm{i}}(0)$ increases by $10 \%$ as shown in figure 20 (e) (black broken line).

It is worthwhile to mention that nonlinear effects in the beta stabilization of ITG turbulence [49] become significant in the central region of these plasmas, where $\beta_{e}$ exceeds $1 \%$ around mid-radius and $2 \%$ in the centre. These nonlinear effects are not included in a linear model like TGLF and their inclusion in the prediction of the central temperatures should be expected to lead to a non-negligible increase.

While the core ion temperature peaking cannot be reproduced entirely by the TGLF modelling - the experimental profile is more peaked in the D plasma - the general trend of higher ion heat diffusivity in $\mathrm{H}$ compared to $\mathrm{D}$ is reproduced. The individual magnitude of fast-ion content contributes significantly to the difference in heat transport.

\subsection{GENE}

Using the experimental parameters of the high power discharges (ID 3, 7) presented in section 2 as input, linear and non-linear gyro-kinetic simulations with the GENE (Gyrokinetic Electromagnetic Numerical Experiment) code [21,22] have been performed. The main goal is to study the effect of the different fast ion pressure in $\mathrm{H}$ and $\mathrm{D}$ plasmas on the plasma turbulence. GENE solves the gyrokinetic Vlasov equations [50,51] coupled with the Maxwell equations within a $\delta f$ approximation and using a set of field aligned coordinates $\left\{x, y, z, v_{\|}, \mu\right\} . z$ is the coordinate along the magnetic field line, $x$ is the radial coordinate, $y$ is the binormal coordinate, $v_{\|}$is the parallel velocity and $\mu$ is the magnetic momentum. The simulations are carried out in the local limit using realistic geometry (reconstructed from numerical equilibrium files provided by equilibrium solvers), collisions (using a 
Landau-Boltzmann operator), finite- $\beta$ effects (considering both $B_{\perp}$ and $B_{\|}$fluctuations), external flow shear $\gamma_{E \times B}$ effects, kinetic ions, kinetic electrons (with the realistic mass ratio) and kinetic fast ions introduced by the NBI heating. For the fast ions, an equivalent Maxwellian distribution function has been used. No impurities have been considered in the simulations, the experimental values of $Z_{\text {eff }}$ of these discharges being low $\left(Z_{\text {eff }}<1.5\right)$. Typical grid parameters in the nonlinear simulations were as follows: perpendicular box sizes $\left[L_{x}, L_{y}\right] \approx[190,200] \rho_{s}$, phase-space grid discretization $\left[n_{x}, n_{y}, n_{z}, n_{v \|}, n_{\mu}\right] \approx[384,64,40,48,16]$ and $0.03 \lesssim k_{y} \rho_{s} \lesssim 1.92$. The choice of $\left(k_{y} \rho_{s}\right)_{\min }=0.03$ corresponds in these cases to a minimum toroidal mode number $n_{0}=2$. In all the simulations, the nonlinear fluxes are mainly related to the electrostatic potential $\phi$ fluctuations while the contribution from the parallel magnetic potential $A_{\|}$fluctuations are smaller $(\sim 5 \%$ of the total fluxes).

The gyrokinetic analysis has been carried out at $\rho_{\text {tor }}=0.3$ corresponding to $\rho_{\text {pol }}=0.43$, where there is a difference between the fast-ion concentration and the $T_{i}$ profiles of $\mathrm{H}$ and $\mathrm{D}$ plasmas.

Linear GENE : The linear growth rates $\gamma\left(\rho / c_{\mathrm{s}}\right)$ of ITG (ion temperature gradient) modes are calculated for the deuterium plasma (ID 3) parameters with mass number $A=2$ (solid lines) and $A=1$ (dashed lines). The sound speed $c_{\mathrm{s}}$ is calculated with the corresponding mass for $\mathrm{H}$ and D. The results are shown in figure 21, The electrostatic simulation without collisions (green) shows no systematic mass dependence in the normalized growth rates, thereby, reproducing a pure gyro-Bohm scaling. It should be noted that only for this simplified case the gyro-Bohm scaling is reproduced in the gyrokinetic simulation. With the inclusion of previously neglected physics mechanisms the simulations deviate more and more from the ideal gyro-Bohm scaling. Taking collisions into account in the simulation (purple) results in $5-10 \%$ higher growth rates for hydrogen. This effect can be expected from the increased impact of collisions in D on the trapped electron contribution to the ITG [45,52]. This increases to $10-20 \%$ when also including electromagnetic effects (black). Although, this goes in the direction of the experimental observations it is not enough to explain its magnitude $\chi_{\mathrm{i}, \mathrm{H}}=2.3 \chi_{\mathrm{i}, \mathrm{D}}$ as seen in figure 15 (f) at $\rho_{\text {tor }}=0.3$ or in normalized units $q_{\mathrm{i}, \mathrm{H}} / q_{\mathrm{i}, \mathrm{H}, \mathrm{gB}}=2.5 q_{\mathrm{i}, \mathrm{D}} / q_{\mathrm{i}, \mathrm{D}, \mathrm{gB}}$. However, when taking fast ions into account the normalized growth rates drop significantly and show a strong mass dependence opposite to gyro-Bohm for the large scales as seen in figure 21 (blue and red triangles). This mass dependence stems from the different fast-ion content in $\mathrm{H}$ and $\mathrm{D}$ where $n_{\text {fast,H }}<n_{\text {fast,D }}$ as discussed in section 2.2.5. How the growth rates translate into heat fluxes is discussed with the help of non-linear gyrokinetic simulations in the next section.

Non-linear GENE : For both D and H plasmas (ID 3 and 7), simulations with and without fast ions are carried out and compared to the experiment. For the D case, a simulation using the fast-ion parameters (except for the mass) of the $\mathrm{H}$ plasmas has also been carried out in order to establish the role of the fast-ion population in explaining the experimental differences in $T_{i}$ observed between $\mathrm{H}$ and $\mathrm{D}$ plasmas. The results are reported in Table $\amalg$ and figure 22 where the heat flux in gyro-Bohm units is plotted against the normalized ion temperature gradient length $R / L_{\mathrm{Ti}}$.

The simulations with hydrogen as main-ion species match the experimental heat flux extremely 


\begin{tabular}{ccrrc}
\hline $\exp$ ID & $\mathrm{A}$ & $W_{\text {fast }}$ & $R / L_{\mathrm{Ti}}$ & \multicolumn{1}{c}{$q_{\mathrm{i}} / q_{\mathrm{i}, \mathrm{gB}}$} \\
\hline 7 & 1 & 0 & 4.69 & $18.0 \pm 1.0$ \\
7 & 1 & $W_{\mathrm{f}, 7}$ & 4.69 & $14.0 \pm 1.0$ \\
7 & 1 & $W_{\mathrm{f}, 7}$ & 3.90 & $0.5 \pm 0.05$ \\
3 & 2 & 0 & 7.10 & $51.5 \pm 4.0$ \\
3 & 2 & $W_{\mathrm{f}, 3}$ & 7.10 & $17.2 \pm 2.0$ \\
3 & 2 & $W_{\mathrm{f}, 3}$ & 5.00 & $1.0 \pm 0.04$ \\
3 & 2 & $W_{\mathrm{f}, 7}$ & 7.10 & $31.0 \pm 2.0$ \\
3 & 2 & $W_{\mathrm{f}, 7}$ & 5.00 & $3.6 \pm 0.6$ \\
\hline
\end{tabular}

TABle iI: Heat fluXes for the Different nON-Linear Gene simulations. $W_{\mathrm{f}, \mathrm{n}}$ IS the experimental Fast-ion CONTENT of the plasma with ID $n$ as in Table

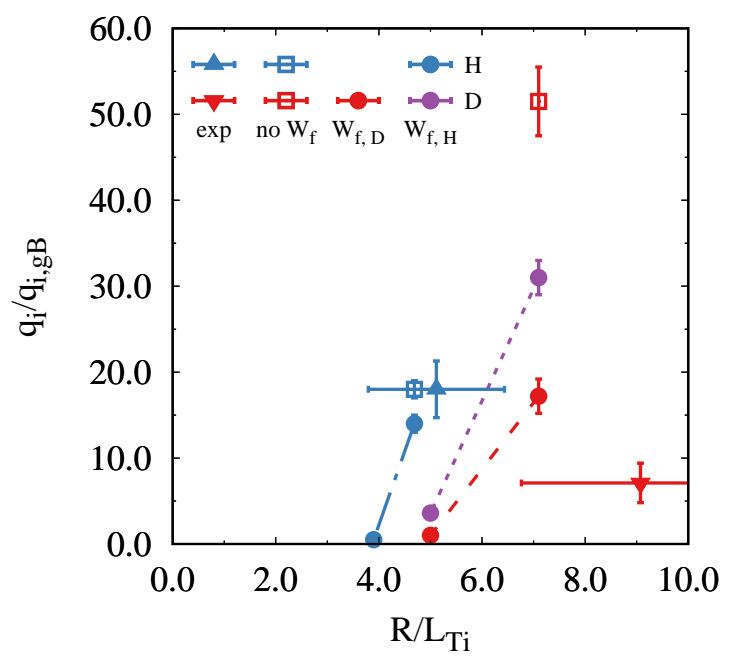

FIG. 22: Normalized heat fluxes predicted by non-linear GENE simulations for different isotopes, fast-ion content and gradient lengths at $\rho_{\mathrm{tor}}=0.30$ or $\rho_{\mathrm{pol}}=0.43$. The experimental points are from pair (ID 3, 7). All combinations of input parameters for the simulations are also listed in Table II. 

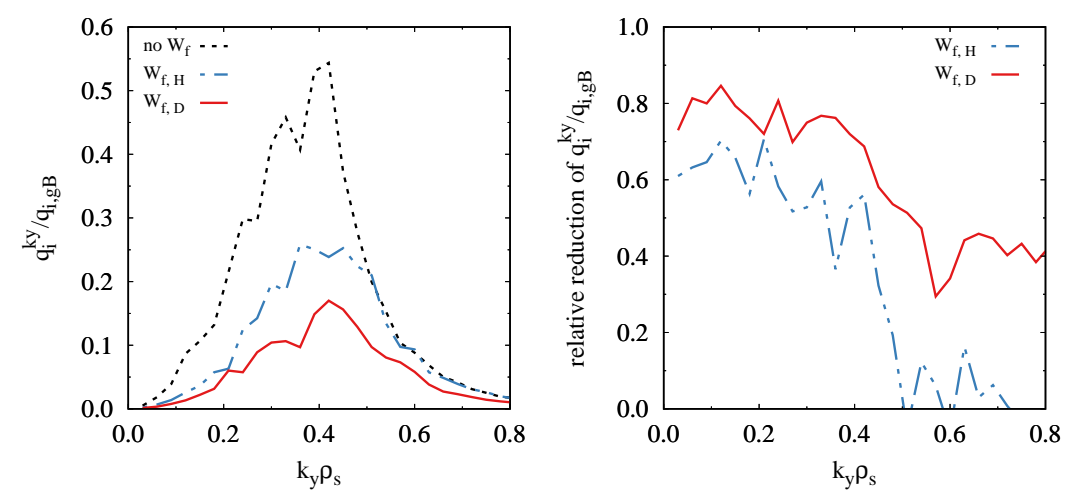

FIG. 23: Non-linear ion heat flux spectra for different fast-ion content (a) and the reduction of heat fluxes relative to the no fast ion simulation $\left(\left(q_{\text {nofast }}-q_{\text {fast }}\right) / q_{\text {nofast }}\right)(b)$. The heat fluxes are negligible for $k_{y} \rho_{s}>0.8$ and therefore, not plotted.

well (blue points). This is true for the run with fast ions as well as the one without fast ions. The impact of an additional fast-ion species is small for the low fast-ion content found in the hydrogen plasma. There is a difference in $R / L_{\mathrm{Ti}}$ between the GENE input and the experimental value. As input we use the gradient obtained from the spline fits shown in figure 9 (c) which is limited by the regularisation of the fit function. The experimental gradient length and its uncertainty is obtained locally on $4 \mathrm{~cm}$ intervals by a linear fit to $\ln T_{\mathrm{i}}$ and tends to be larger due to the missing regularisation.

The simulation without fast ions and deuterium as main-ion species (red open square) shows heat fluxes nearly an order of magnitude above the experimental ones. It is opposite to the experimental trend, which shows lower heat fluxes for steeper gradients in D compared to $\mathrm{H}$.

Including fast ions has a strong stabilising effect on the ITG turbulence in the deuterium cases. With the experimental $W_{\text {fast,D }}$ the gyrokinetic heat fluxes are within a factor of 2 of the experimental $q_{\mathrm{i}} / q_{\mathrm{i}, \mathrm{gB}}$. While, when using the lower $W_{\mathrm{fast}, \mathrm{H}}$, even if the turbulence is strongly suppressed, the heat flux is 4 times the experimental one. The profile stiffness is reduced considerably in the simulation when including fast-ions in D which is similar to observations in JET L-modes [12]. A comparable experimental scan to determine the profile stiffness in the H-mode core is challenging as one would need to vary the heat fluxes while keeping the pedestal as well as the fast-ion content constant. Additionally, at power levels required to observe a significant impact of fast ions, there are no heat sources available for comparison which do not generate fast ions as well. The pedestal can be kept constant in $\mathrm{D}$ while changing the core ion heat flux by comparing plasmas with onand off-axis NBI heating. However, even then a strong correlation of ion heat flux and fast-ion content cannot be avoided and the profile stiffness is reduced by the fast ions [43].

The impact of fast ions in the simulations is also visible in the heat flux spectra per $k_{y} \rho_{s}$ which are illustrated in figure 23 (a) and (b). Adding fast ions in the simulation reduces the heat flux by the larger structures $k_{y} \rho_{s}<0.4$ up to $80 \%$ (b). For the relatively large fast-ion content, in the $\mathrm{D}$ case, the heat flux is reduced for all scales.

A difference of measured and simulated heat fluxes in D is present and might be due to uncertainties in the data not considered for the error bars, in particular, the q profile in the core. Another source for discrepancies are simplified physics mechanisms in the model, e.g. by using a Maxwellian 
distribution for fast ions instead of a more realistic slowing down distribution [53]. This is a potential explanation why for $\mathrm{H}$ - where fast ions have little impact - model and experiment agree better than for the simulations in D. However, the simulations done in $\mathrm{H}$ and those in $\mathrm{D}$ are also different in terms of $T_{\mathrm{e}} / T_{\mathrm{i}}$ and the total $\beta$, which could be a source for deviations.

The main conclusion remains robustly above the uncertainties in the modelling: The fast-ion stabilization of ITG turbulence [12,41-43] is important in these plasmas and the difference in fastion population between $\mathrm{H}$ and $\mathrm{D}$ results in reduced heat transport in $\mathrm{D}$ compared to $\mathrm{H}$ plasmas.

\section{Discussion and conclusion}

The energy confinement in fusion plasmas shows a strong dependence on the isotope mass. This was confirmed in our study and multiple factors were found to be relevant for this conclusion. In Hmode the edge transport barrier is a crucial element for the energy confinement, this remains true for different isotopes. Disentangling the co-dependency of core transport and the edge confinement was found to help identify and quantify different contributions to the mass dependence of energy confinement.

For low plasma triangularity the pedestal in $\mathrm{H}$ is more prone to deteriorating effects than in D. In particular, increased gas puffing can lead to a $50 \%$ loss of confinement in $\mathrm{H}$ which is not observed in D. Reasons for this are likely related to edge particle transport in connection with the pedestal stability. The proximity to the LH-transition is probably not an issue. Although, discharges with similar power levels are naturally closer to the LH-transition in hydrogen, because $P_{\mathrm{LH}}^{\mathrm{H}}>P_{\mathrm{LH}}^{\mathrm{D}}$ [54 56], in this study the heating power is well above the threshold with $P_{\text {heat }}>2 P_{\mathrm{LH}}$ also for the hydrogen plasmas.

While a discussion on the underlying edge dynamics is crucial to complete the physics picture, this paper focuses on the core transport. Increasing the plasma triangularity in $\mathrm{H}$ allows us to match the pedestal properties of corresponding D plasmas with comparable heat and particle sources. This was particularly important to extend the study to higher heating powers. At low triangularity the hydrogen plasmas are severely limited in the achievable density resulting in intolerable shine through losses of the NBI. This limitation is overcome at higher triangularity. Another aspect is the matching of rotation profiles for $\mathrm{H}$ and $\mathrm{D}$ with the same NBI power levels, which became possible because the momentum confinement did recover with the higher triangularity. However, the results of quasilinear modelling with TGLF show no significant impact of the rotation profiles on heat transport in the presented plasma regime.

Based on comparisons with matched pedestal parameters, the plasma core is investigated. The fast-ion slowing down is found to be the dominating mass dependent mechanism in explaining the experimental observations. When aiming for isotope purity by using D-NBI into D and H-NBI into $\mathrm{H}$, the fast-ion content in the $\mathrm{H}$ plasmas will naturally be lower. The contribution from slowing down is often enhanced by present day NBI systems which are restricted to lower acceleration voltages for $\mathrm{H}$.

Plasmas with relatively low heating power - thereby a fast-ion content which has a small impact 
on turbulent transport - show similar core transport when the plasma rotation is matched. This is observed regardless of the isotope mass. This result is consistent with observations previously made in L-mode [4]. When the fast-ion content in the plasma becomes more dominant, for the presented ASDEX Upgrade discharges this means $W_{\text {fast }} / W_{\text {th }}>1 / 3$, the local transport in $\mathrm{D}$ plasmas is reduced and the confinement increases. In $\mathrm{H}$ plasmas at similar power levels, the fastion content will not reach these levels, effectively resulting in higher transport and lower energy confinement.

This experimental observation is consistent with ASTRA modelling with TGLF and with gyrokinetic simulations with GENE. The ITG growth rates from linear GENE calculations show a strong reduction with increasing fast-ion content. Since the fast-ion content is correlated with the isotope mass, the $\mathrm{D}$ plasma shows lower growth rates than the $\mathrm{H}$ plasma, which is opposite to the mass dependence expected for gyro-Bohm like transport. In non-linear GENE simulations with kinetic fast ions the experimental heat fluxes are reproduced within a factor of 2. Also the empirical level of necessary fast-ion content to observe an impact on confinement is confirmed. Simulations including collisions, finite- $\beta$ effects, external flow shear $\gamma_{E \times B}$ effects, kinetic ions and kinetic electrons, but without fast ions match the experimental hydrogen heat fluxes well. Simulations with only these physics ingredients yield heat fluxes one order of magnitude above the experimental ones of the deuterium plasma, while providing a decent match when the turbulence stabilisation by fast-ions is taken into account.

Effectively, this results in a higher stiffness of $T_{\mathrm{i}}$ in $\mathrm{H}$, because in contrast to $\mathrm{D}$ the stabilising contribution of the fast ions is missing. A similar observation was made in JT60-U where the profile stiffness was found to be higher in $H$ than in $D$ [11]. Considering, the fast-ion content in $H$ was substantially lower than in D - like in AUG - one could expect that the turbulence stabilisation by fast-ions does also explain the observations made in JT60-U.

Since fast ions will become more important in fusion plasmas [57], the mass dependence of the fast-ion slowing down time will play an important role in translating observations from pre-nuclear plasma operation to the nuclear phase. Therefore, the inclusion of fast-ion effects in theoretical models is essential to improve their predictive capabilities for fusion plasmas.

This work has been carried out within the framework of the EUROfusion Consortium and has received funding from the Euratom research and training programme 2014-2018 and 2019-2020 under grant agreement No 633053. The views and opinions expressed herein do not necessarily reflect those of the European Commission.

[1] CHALLIS, C. D. et al., Nuclear Fusion 55 (2015) 53031.

[2] DUNnE, M. G. et al., Plasma Physics and Controlled Fusion 59 (2017) 25010.

[3] KOBAYASHI, T. et al., Scientific Reports 915913.

[4] SCHNEIDER, P. A. et al., Nuclear Fusion 57 (2017) 66003.

[5] DELABIE, E. et al., Preliminary interpretation of the isotope effect on energy confinement in Ohmic discharges in JET-ILW, in 44th EPS Conference on Plasma Physics, page P4.159, Geneva, 2017, European Physical Society.

[6] KUPRIENKO, D. V. et al., Plasma Physics Reports 45 (2019) 1128. 
[7] MAGGI, C. F. et al., Nuclear Fusion 59 (2019) 76028.

[8] BONANOMI, N. et al., Nuclear Fusion 59 (2019) 126025.

[9] LAGgneR, F. M. et al., Physics of Plasmas 24 (2017) 56105.

[10] WEISEN, H. et al., Journal of Plasma Physics 86 (2020) 905860501.

[11] URANO, H. et al., Nuclear Fusion 53 (2013) 83003.

[12] BONANOMI, N. et al., Nuclear Fusion 59 (2019) 96030.

[13] TERRY, P. W., Review of Modern Physics 72 (2000) 109.

[14] MANINI, A. et al., Nuclear Fusion 46 (2006) 1047.

[15] TARDINI, G. et al., Nuclear Fusion 47 (2007) 280.

[16] SCHNEIDER, P. A. et al., Nuclear Fusion 53 (2013) 73039.

[17] STAebleR, G. M. et al., Physics of Plasmas 14 (2007) 55909.

[18] STAEBLER, G. M. et al., Nuclear Fusion 57 (2017) 66046.

[19] PEREVERZEV, G. V. et al., IPP Report 5/98 (2002).

[20] FABLE, E. et al., Plasma Physics and Controlled Fusion 55 (2013) 124028.

[21] JENKO, F. et al., Physics of Plasmas 7 (2000) 1904.

[22] GöRLER, T. et al., Journal of Computational Physics 230 (2011) 7053.

[23] DENK, S. S. et al., Plasma Physics and Controlled Fusion 60 (2018) 105010.

[24] KURZAN, B. et al., Review of Scientific Instruments 82 (2011) 103501.

[25] WILlensDorfer, M. et al., Review of Scientific Instruments 83 (2012) 23501.

[26] MCDERMOTT, R. M. et al., Review of Scientific Instruments 88 (2017) 73508.

[27] CAVEDON, M. et al., Review of Scientific Instruments 88 (2017) 43103.

[28] BERNERT, M. et al., Review of Scientific Instruments 85 (2014) 033503.

[29] GEIGER, B. et al., Review of Scientific Instruments 84 (2013) .

[30] POTZEL, S. et al., Journal of Nuclear Materials 463 (2015) 541.

[31] DUnne, M. G. et al., Plasma Physics and Controlled Fusion 59 (2017) 14017.

[32] SUTTROP, W. et al., Plasma Physics and Controlled Fusion 42 (2000) A97.

[33] DE VRIES, P. C. et al., Plasma Physics and Controlled Fusion 48 (2006) 1693.

[34] STIX, T. H., Plasma Physics 14 (1972) 367.

[35] WEILAND, M. et al., Nuclear Fusion 58 (2018) 82032.

[36] ITER Physics Expert Group, Nuclear Fusion 39 (1999) 2137.

[37] PANKIN, A. et al., Computer Physics Communications 159 (2004) 157.

[38] BRESLAU, J. et al., TRANSP, [Computer Software] \url\{https://doi.org/10.11578/dc.20180627.4\}, 2018.

[39] GEIGER, B. et al., Nuclear Fusion 55 (2015) 83001.

[40] Jansen Van Vuuren, A. et al., Review of Scientific Instruments 90 (2019) 103501.

[41] CITRIN, J. et al., Phys. Rev. Lett. 111 (2013) 155001. 
[42] Di Siena, A. et al., Nuclear Fusion 58 (2018) 54002.

[43] RYTER, F. et al., Nuclear Fusion 59 (2019) 96052.

[44] ANGiOni, C. et al., Plasma Physics and Controlled Fusion 51 (2009) 124017.

[45] ANGiOni, C. et al., Physics of Plasmas 25 (2018) 82517.

[46] REISNER, M. et al., Nuclear Fusion 60 (2020) 82005.

[47] SYNAKOWSKI, E. J. et al., Phys. Rev. Lett. 78 (1997) 2972.

[48] SHIRAI, H. et al., Nuclear Fusion 39 (1999) 1713.

[49] CITRIN, J. et al., Nuclear Fusion 54 (2014) 23008.

[50] FRIEMAN, E. A. et al., The Physics of Fluids 25 (1982) 502.

[51] BRIZARD, A. J. et al., Rev. Mod. Phys. 79 (2007) 421.

[52] NAKATA, M. et al., Phys. Rev. Lett. 118 (2017) 165002.

[53] Di Siena, A. et al., Physics of Plasmas 25 (2018) 42304.

[54] RYTER, F. et al., Plasma Physics and Controlled Fusion 58 (2016) 14007.

[55] MAGgI, C. F. et al., Plasma Physics and Controlled Fusion 60 (2018) 14045.

[56] PLANK, U. et al., Nuclear Fusion 60 (2020) 74001.

[57] WENNINGER, R. et al., Nuclear Fusion 55 (2015) 63003. 